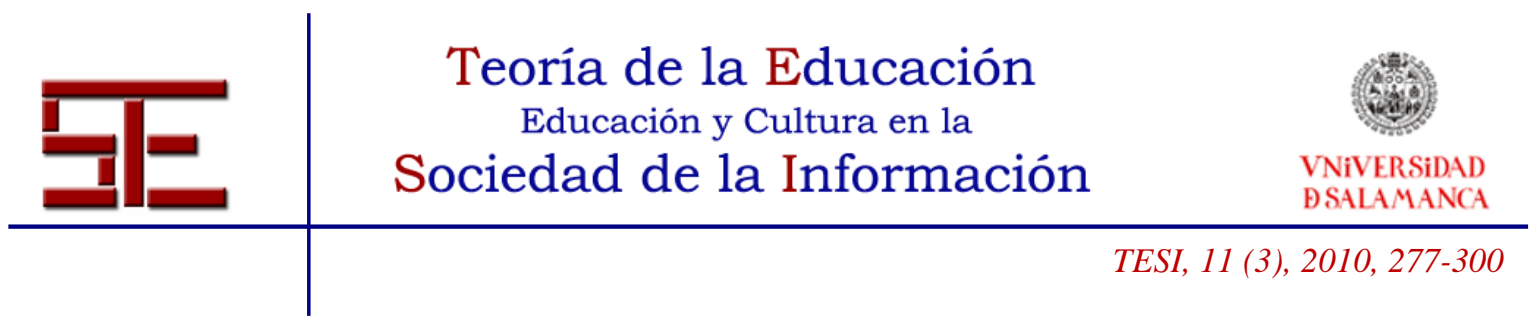

\title{
HERRAMIENTAS PARA EL APRENDIZAJE COLABORATIVO: UNA APLICACIÓN PRÁCTICA DEL JUEGO DE ROL
}

Resumen: El juego de rol es una herramienta de participación activa del alumno que facilita el aprendizaje cooperativo y que puede ser más efectiva que los métodos tradicionales para la formación en competencias. Esta técnica es esencial para compatibilizar la teoría y la práctica que se requiere para la adaptación de las asignaturas al EEES, en especial las que pertenecen al ámbito de Ciencias Sociales. Por ello, el objetivo de este trabajo es demostrar el efecto y la utilidad del juego de rol en el ámbito de la Organización de Empresas. En concreto, se analiza y explica el diseño y la puesta en práctica de esta herramienta en al aula en la asignatura de 'Dirección de Recursos Humanos'. Su utilización durante varios cursos académicos nos ha mostrado que es una herramienta útil, que facilita el aprendizaje del alumno y el desarrollo de capacidades como el trabajo en grupo, la resolución de problemas y conflictos, la toma de decisiones y la dirección de sistemas complejos.

Palabras clave: juego de rol; dirección de Recursos Humanos; aprendizaje colaborativo

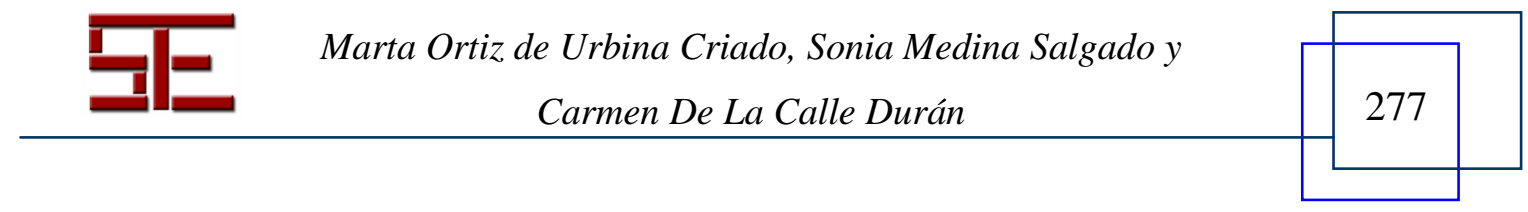




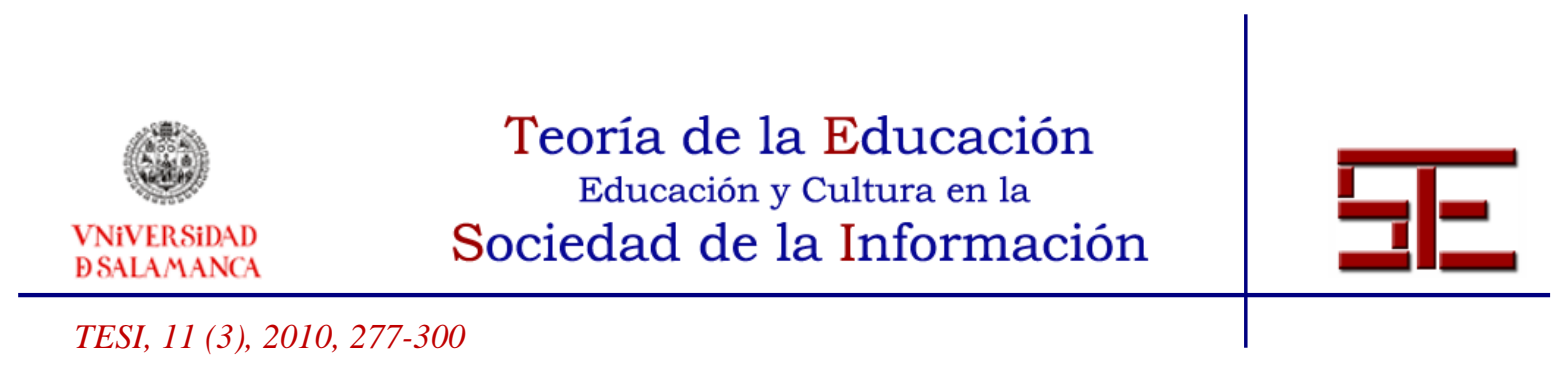

\section{TOOLS FOR COLABORATIVE LEARNING: A ROLE-PLAYING PRACTICE}

Abstract: Role-playing is an active participation tool that facilitates cooperative learning. It has also proved to be more effective in developing competencies than traditional methods. This technique is essential to make theory and practice compatible as is required in order to adapt subjects to the new education system based on the Bolonia's agreement, especially in Social Sciences disciplines. Consequently, the objective of this paper is to show the effect and use of role-playing applied to management area. Therefore, we analyze and design the role-playing, putting it in practice in the classroom in Human Resource Management subject of different academic degrees. To conclude, this paper has shown the importance of role-playing as a learning tool and development of skills like work cooperation, problem and conflict solving, decision making, and managing complex systems.

Keywords: role-playing; human resource management; cooperative learning.

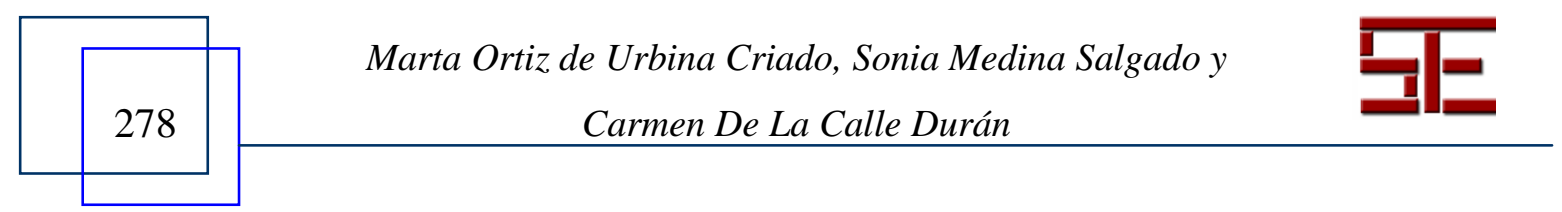




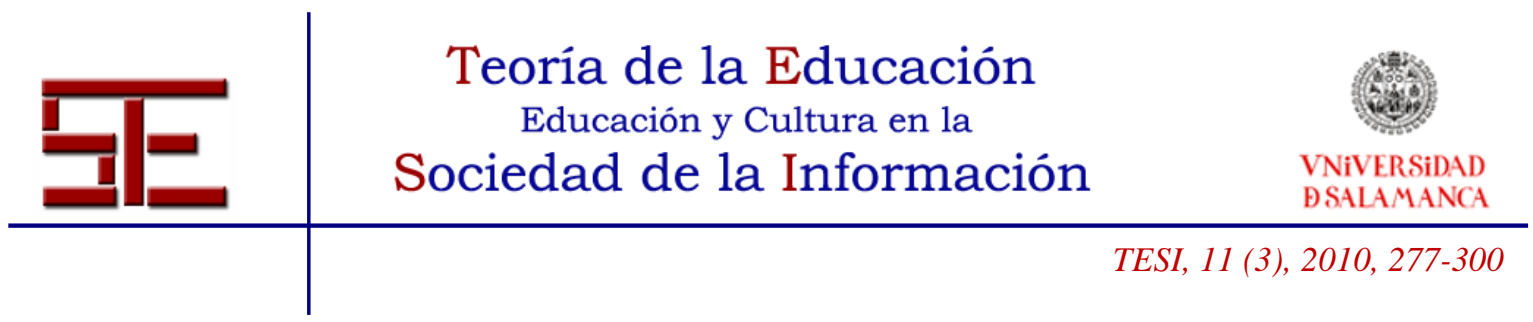

\section{HERRAMIENTAS PARA EL APRENDIZAJE COLABORATIVO: UNA APLICACIÓN PRÁCTICA DEL JUEGO DE ROL}

Fecha de recepción: 25/07/2010; fecha de aceptación: 18/10/2010; fecha de publicación: 30/11/2010

Marta Ortiz de Urbina Criado

marta.ortizdeurbina@urjc.es

Universidad Rey Juan Carlos

Sonia Medina Salgado

sonia.medina@urjc.es

Universidad Rey Juan Carlos

Carmen De La Calle Durán

carmen.delacalle@urjc.es

Universidad Rey Juan Carlos

\section{1.- INTRODUCIÓN}

En el nuevo sistema educativo europeo, basado en el acuerdo de Bolonia, se hace necesario reflexionar sobre la mejor forma de lograr tanto el aprendizaje individual como el colectivo. El Espacio Europeo de Educación Superior (EEES) pretende impulsar un enfoque más plural de la actividad docente con el fin de que el estudiante tenga más oportunidades para el desarrollo de nuevas competencias que permitan al alumno ser el protagonista en la búsqueda del conocimiento (De Miguel, 2006; Aymerich ; Gras, 2009). Para ello hay que introducir cambios en la función docente de forma que el profesor pase a tener un papel de planificador, de facilitador, de guía y moderador y que el alumno aprenda a ser autónomo y tenga una actitud participativa, grupal y comprometida con los procesos que se llevan a cabo en el aula (Parra, 2002; Inda et al., 2008). Por todo ello, uno de los retos de la universidad del siglo XXI es la

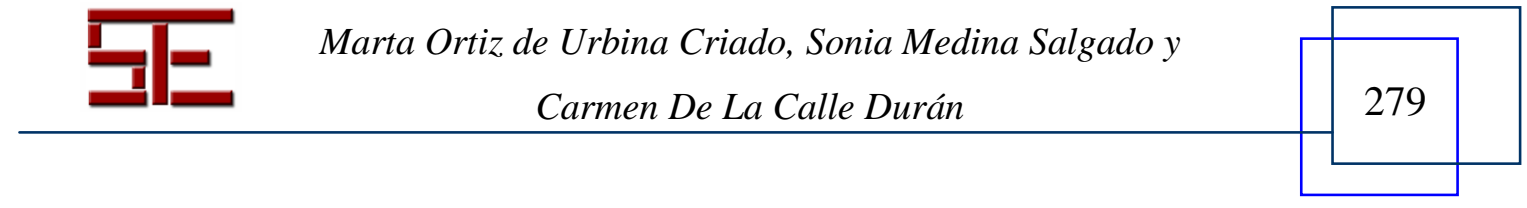




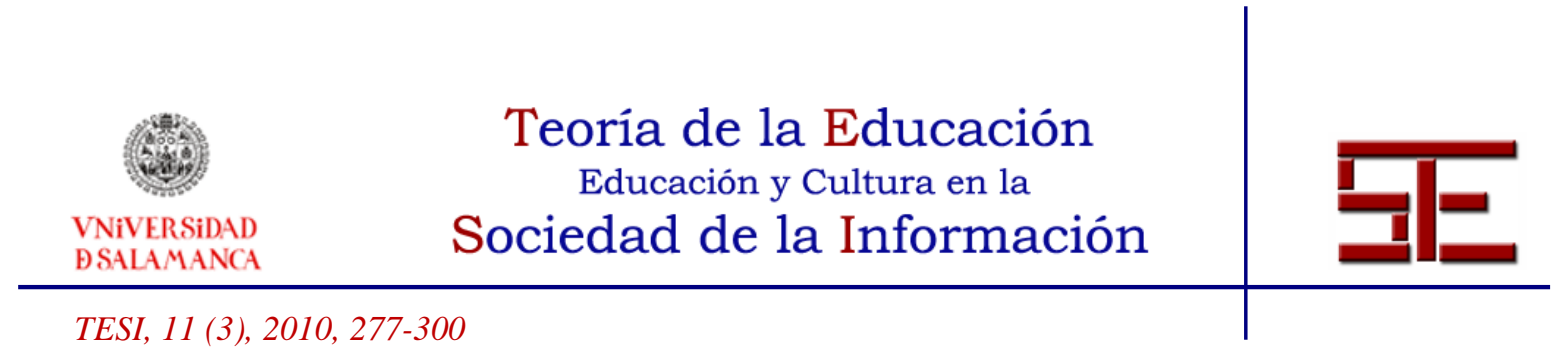

renovación metodológica. La metodología docente mayoritaria en el entorno universitario ha sido, hasta el momento, la clase magistral, pero los cambios acelerados que se están viviendo en las últimas décadas a nivel socio cultural y tecnológico han llevado a ampliar y readaptar las metodologías docentes a las nuevas demandas de un entorno profesional cada vez más exigente para los titulados superiores (Aymerich ; Gras, 2009, 1). Ello ha tenido como consecuencia la proliferación de iniciativas para cambiar el proceso enseñanza-aprendizaje centradas, en su mayor parte, en poner el acento en la persona que aprende más que en la que enseña y dando más importancia a la acción de apoyar al alumno en el proceso de adquisición de conocimientos que a la tarea de trasmitirlos (Marzano, 1991; Inda et al., 2008).

A través de nuestra experiencia docente hemos observado que la clase magistral no cubre este tipo de habilidades, ni siquiera el trabajo práctico de carácter individual. Ello nos ha llevado a plantearnos la importancia de potenciar el aprendizaje cooperativo y experiencial, especialmente, en el ámbito universitario. Para conseguir ese reto hay diversas técnicas que pueden utilizarse en el aula y que sirven para que el alumno aprenda a aprender, aprenda a trabajar en grupo y aprenda a analizar problemas y a tomar decisiones tanto individuales como en grupo. Una de esas técnicas, todavía poco utilizada en las universidades españolas, es el juego de rol (role-playing).

El juego de rol es una herramienta de participación activa del alumno, que facilita el aprendizaje cooperativo y que, además, demuestra ser más efectiva que los métodos tradicionales para la formación en competencias. Estas competencias, además de ser muy necesarias para el desarrollo profesional de los alumnos, se requieren para la adaptación al EEES, en el que actualmente la universidad española se encuentra inmersa. El paradigma de "aprender haciendo" es especialmente difícil de lograr en disciplinas como las Ciencias Sociales que no tienen laboratorios o trabajo de campo. Por ello, esta técnica es esencial para compatibilizar la teoría y la práctica que se requiere para la adaptación de las asignaturas al nuevo sistema educativo EESS basado en el acuerdo de Bolonia. En consecuencia, el objetivo de este trabajo es demostrar la utilidad del juego de rol en el campo de la Organización de Empresas. En concreto, se analiza y explica el diseño (metodología) y la puesta en práctica de esta herramienta en el aula. La experiencia que se analiza en este trabajo es el resultado de la aplicación durante varios cursos académicos de esta técnica en la asignatura de 'Dirección de Recursos Humanos' impartida en varias titulaciones de la Universidad Rey Juan Carlos: Licenciatura en Administración y Dirección de Empresas, Diplomatura en Turismo y Master Oficial en Relaciones Laborales.

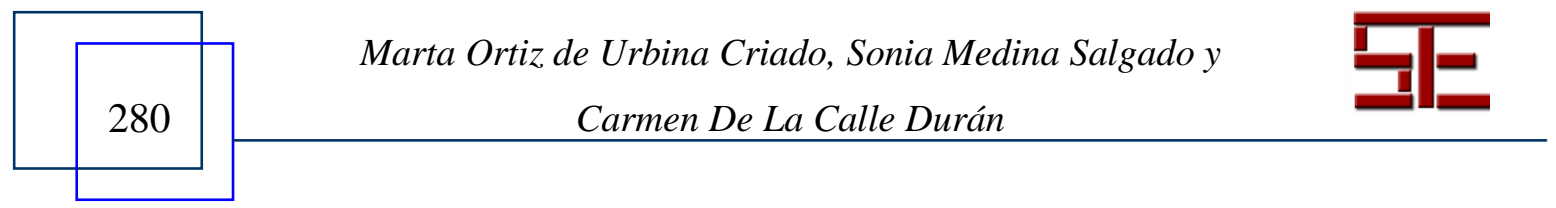




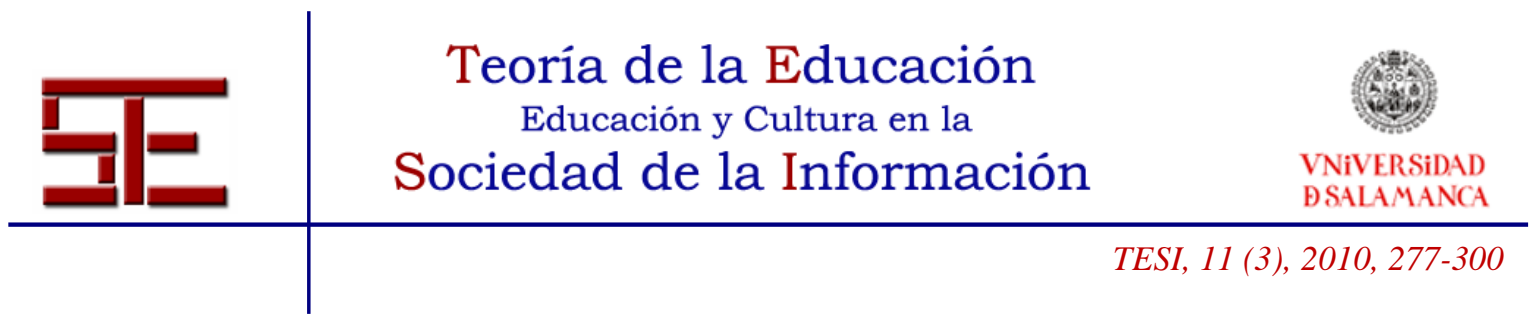

El trabajo se ha estructurado de la siguiente forma: en primer lugar, se ha hecho una revisión de los antecedentes de la metodología del juego de rol que justifica la utilidad de esta herramienta para el aprendizaje cooperativo; a continuación se ha analizado en profundidad una aplicación práctica realizada en el aula para los alumnos de la asignatura de Dirección de Recursos Humanos impartida en diferentes titulaciones en la Universidad Rey Juan Carlos. Por último, se aportan las oportunas conclusiones del mismo.

La principal aportación de este trabajo es mostrar la utilidad del juego de rol como herramienta efectiva para potenciar habilidades como: el trabajo en equipo, la resolución de conflictos, la toma de decisiones y la gestión de otros sistemas complejos, que son esenciales para desenvolverse en el ámbito empresarial actual.

\section{2.- PERSPECTIVA EDUCATIVA DEL JUEGO DE ROL}

La formación de profesionales universitarios con capacidad para ejercer cualquier profesión a nivel directivo se está sometiendo a un proceso de discusión y de cambio. La comunidad educativa europea, a partir del proceso de Bolonia, pone de manifiesto el interés por formalizar un cambio de modelo educativo que evoluciona desde los métodos más clásicos de la clase magistral hacia otros más implicados con los distintos niveles de aprendizaje de los alumnos y con el mejor desarrollo de competencias para el mundo real. Además, la demanda actual de profesionales en todos los campos del conocimiento reclama personas capaces de enfrentarse a situaciones reales y de cambio continuo en las mejores condiciones. Así mismo, en el mundo laboral, en el que el trabajo de equipo es el modo habitual de trabajar, se requieren, cada vez más, empleados capaces de comunicar y aprender unos de otros de un modo efectivo (Yazici, 2004).

Según una encuesta hecha a setenta grandes compañías químicas sobre cuáles debían ser las características que deberían tener los ingenieros químicos y cómo esas características estaban relacionadas con la habilidad de las personas para contribuir a la efectividad y a la misión de un grupo, Jackson y Walters (2000) mostraban que las personas ya no trabajan "aisladas" y que, en una era de tecnología de la comunicación, los equipos afrontan problemas y las personas necesitan desarrollar competencias que les dirijan a mejorar la productividad. Por otro lado, Rakesh Kuhurana (2007), en su libro From Higher Aims to Hired Hands, se lamenta de que las habilidades interpersonales y de comunicación y liderazgo han dejado de ser impartidas en las

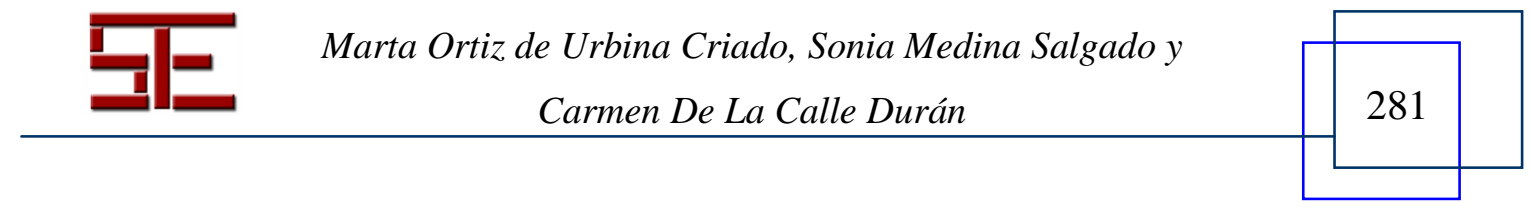




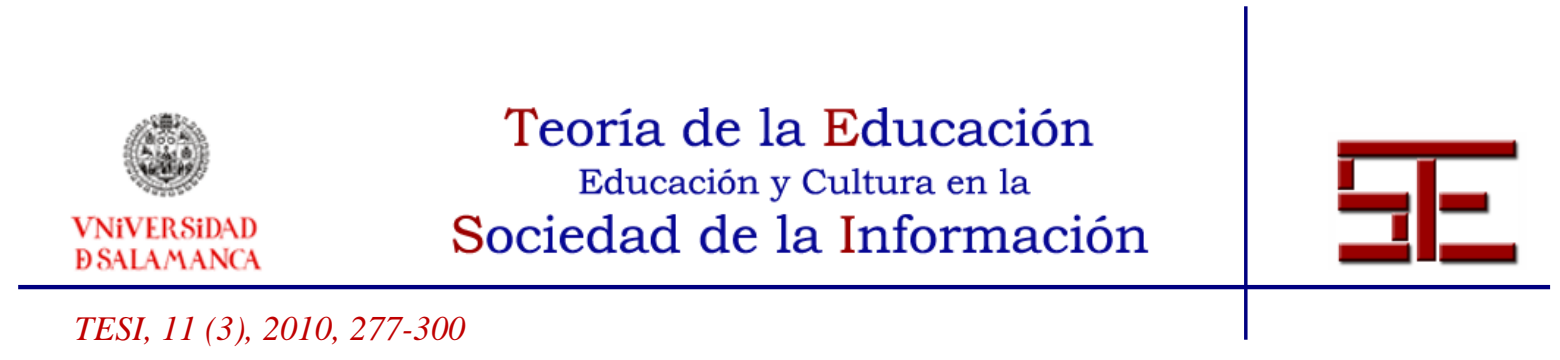

escuelas de negocios norteamericanas en los últimos tiempos. Para él, estos centros han pasado de formar profesionales con unos conocimientos específicos y ciertos códigos de conducta con una idea de servicio a crear directivos dirigidos, casi en exclusiva, a dar beneficios a los accionistas.

Los métodos de enseñanza tradicionales están basados en un aprendizaje pasivo y los enfoques pedagógicos están centrados en el profesor y relacionados con la memorización de conceptos (Sogunro, 2004). Con ellos el alumno escucha, memoriza y aplica los contenidos sin que, en múltiples ocasiones, se haya producido un aprendizaje real que le capacite para actuar en el ejercicio de su profesión o para enfrentarse a nuevas situaciones. Es decir, no facilita ni el pensamiento crítico ni el desarrollo de habilidades de comunicación útiles para el éxito profesional (Muñoz y Huser, 2008). Por otro lado, al modo convencional de enseñanza le falta potencial para cambiar actitudes y comportamientos en los estudiantes ya que no proporciona feedback continuo ni de los profesores ni de los compañeros, es decir, no permite aprender "haciendo" (Ments, 1998).

En el mundo académico en general, y docente en particular, se suele valorar la aplicación de métodos y enfoques pedagógicos variados que permiten mejorar la calidad y la efectividad de la enseñanza (Baruch, 2006). McDonald-Mann (1998) declara, por ejemplo, que la clase magistral es, a menudo, muy necesaria para proporcionar grandes cantidades de información y de teoría, pero, por sí sola, no es suficiente para formar. En definitiva, se evidencia que es necesario incorporar en las aulas aquellos enfoques pedagógicos que mejor resuelvan estas lagunas de los métodos tradicionales.

Uno de esos enfoques es el aprendizaje experiencial (experiential learning) (Wood, 2003) o de learning by doing. Con este enfoque se pretende que el alumno, a través de experiencias concretas, aprenda los conceptos que se le proporcionan al tiempo que aprende a modificar sus conductas para la mejor aplicación de las herramientas aprendidas e incluso para generar nuevas ideas. Es decir, se pretende mejorar el éxito de la enseñanza haciendo que los estudiantes aprovechen las experiencias directas para reflejar, comprobar y generar nuevas ideas (Muñoz y Huser, 2008), permitiendo además desarrollar habilidades fundamentales como la comunicación oral, la investigación, el análisis, el trabajo en equipo (Peterson, 1996).

Por otro lado, el entorno actual demanda formas de trabajo globales, abiertas y de colaboración que requieren motivación, capacidad para aprender de otros y para generar

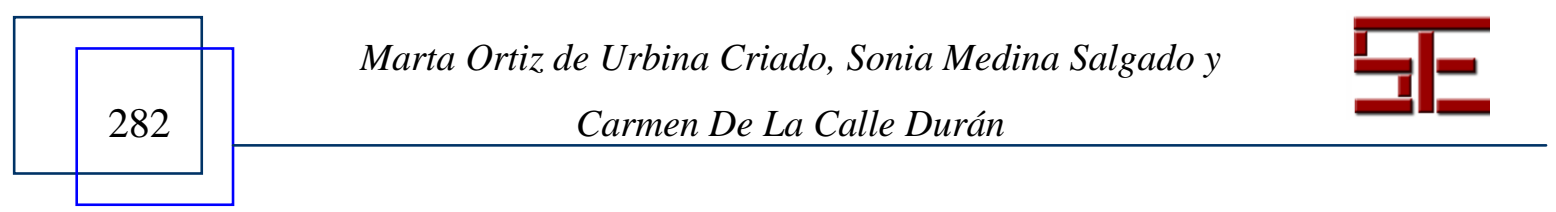




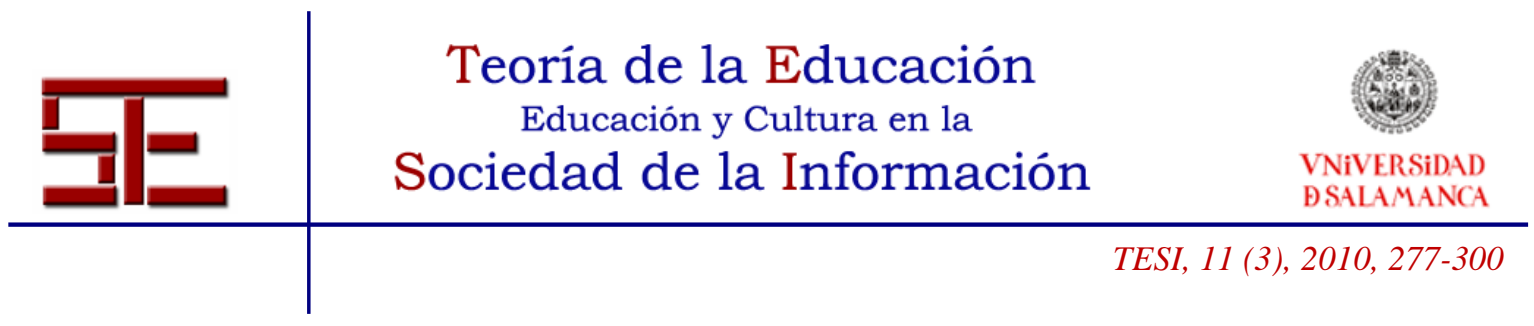

e internalizar ideas en grupo. Otro enfoque útil para poder atender estas nuevas necesidades e incorporarlas en el aula es el aprendizaje colaborativo. Según Johnson y Johnson (1994), para que el aprendizaje colaborativo funcione debemos tener en cuenta cinco componentes esenciales (Padilla et al., 2008, 461):

1.- Interdependencia positiva: Los estudiantes son conscientes de que son un equipo y el trabajo de uno repercute en los demás.

2.- Exigibilidad personal: Cada miembro del grupo debe ser capaz de aportar su conocimiento al grupo y de aprender lo que sus compañeros le aporten, con el fin de beneficiar a todo el grupo: "No vale descansarse sobre los demás".

3.- Interacción positiva cara a cara: Se produce cuando los alumnos comparten sus conocimientos, discuten distintos puntos de vista, ayudan al resto con las dificultades...

4.- Habilidades interpersonales y de grupo: Los estudiantes deben organizar el trabajo y tomar decisiones, manifestando sus dotes de liderazgo, conciliación, etc.

5.- Autoanálisis del grupo: El grupo debe autoanalizarse para saber si su trabajo está siendo efectivo, se alcanzan las metas y se está trabajando en el ambiente adecuado. Esto permite que los miembros del grupo fortalezcan sus habilidades de trabajo y fomenta el compromiso de todos con los objetivos comunes.

Esta perspectiva, en la que encajan muy diversas técnicas (debates, juegos, paneles, etc.), permite a los estudiantes trabajar en grupos ayudándose los unos a los otros (Paulson y Faust, 2008). Consiste, por tanto, en utilizar la formación de grupos de estudiantes de distintos niveles para que aprendan y comprendan mejor una materia interactuando juntos. Este tipo de técnicas hacen que los alumnos compartan información y conocimiento mejorando sus habilidades de comunicación y de grupo y facilitando la confianza en sí mismos sobre su capacidad para definir problemas y aplicar sus conocimientos para resolverlos (Yazici, 2004). En el trabajo de Inda et al. (2008) se pone de manifiesto la existencia de claras diferencias en el rendimiento final cuando se emplean metodologías que implican más al alumno. Sin embargo, aunque estas metodologías no son algo nuevo, las Universidades parecen haber olvidado importantes aspectos pedagógicos de la docencia (Ibáñez-Martín, 1990; Buendía y Olmedo, 2000; Inda et al., 2008) en favor de los conocimientos en sí mismos.

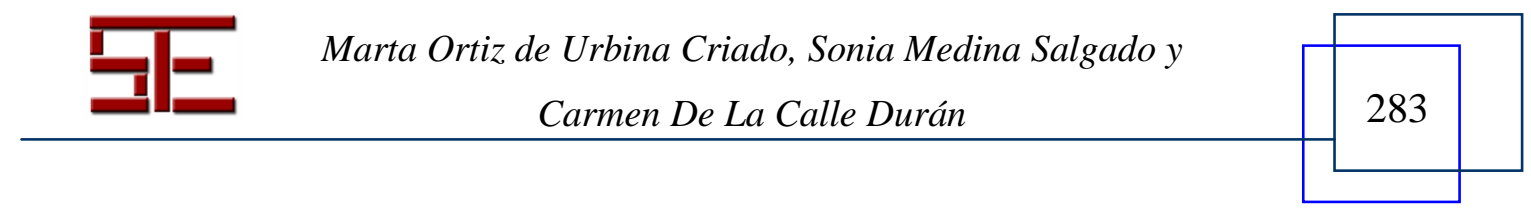




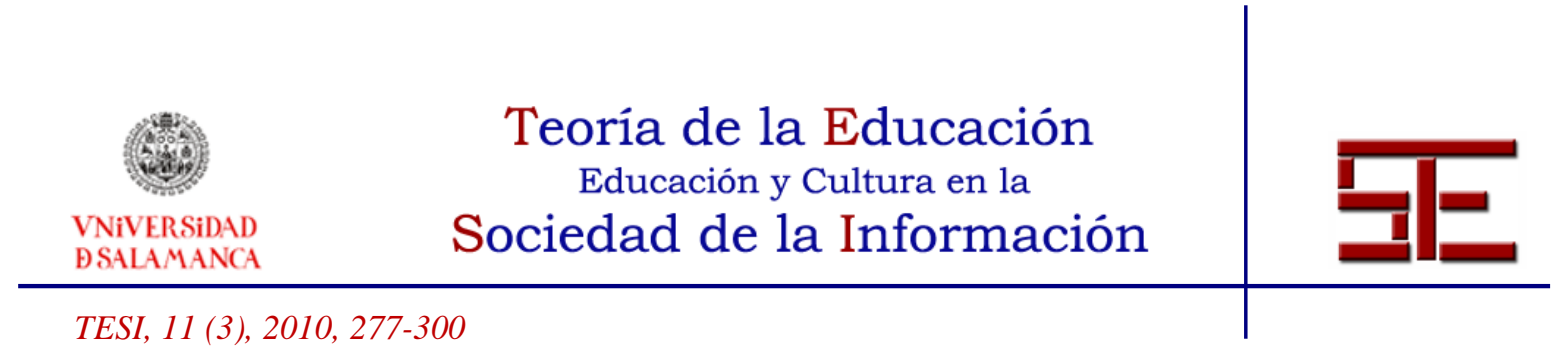

Dados los enfoques de aprendizaje anteriormente descritos, se puede decir que el juego de rol es una técnica de enseñanza-aprendizaje mediante la que de un modo colaborativo los alumnos aprenden jugando y experimentando y el profesor enseña reforzando el aprendizaje. Los juegos pueden ayudar a desarrollar cualidades y/o habilidades psicológicas, cognitivas, físicas y/o sociales, por lo que se han ido incorporando poco a poco a las aulas como complementos curriculares y mediadores del proceso de aprendizaje (Valiño, 2002). En el modelo de aprendizaje propuesto, los estudiantes pueden aprender de la interacción con el resto del grupo y del acercamiento de posturas, puesto que los miembros del grupo dependen unos de otros, se ayudan entre sí y asumen la responsabilidad del éxito o el fracaso común (Jong et al., 2006). Siguiendo la tesis de Piaget y Inhelder (1971), las actividades colaborativas permiten al individuo llegar a dominar tareas que antes era incapaz de realizar de manera individual. Estas actividades contribuyen a crear, entre los participantes, el desequilibrio cognitivo necesario para que se den los procesos de asimilación y acomodación en la construcción del conocimiento, ayudando con ello a desarrollar la habilidad cognitiva individual de cada uno de ellos (Padilla et al., 2008)

El juego de rol, como técnica de aprendizaje, tiene su origen en la representación teatral, sin embargo, su utilidad va más allá de la mera representación de papeles en el aula. Es una herramienta con la que todos los miembros participan activamente con una simulación en grupo. La simulación nunca logra reproducir de forma completa las circunstancias reales, pero sí permite aproximarse a ella de forma razonable. Especialmente, las simulaciones permiten incluir algunos de los componentes de complejidad que se desean desarrollar, por lo que constituyen un buen sistema para facilitar los aprendizajes orientados a la comprensión y la utilización (Castelló, 2007; Aymerich y Gras, 2009).

Para el desarrollo de esta técnica, el formador-profesor proporciona a los alumnos la información suficiente sobre la situación, el problema o conflicto así como las características más relevantes del papel que van a representar: responsabilidades, carácter, emociones, preocupaciones, objetivos, etc. Esta técnica es muy válida para ayudar a entender un problema y desarrollar la empatía, la posibilidad de ponernos y comprender la situación de los demás, por lo que favorece la cohesión del grupo. Es una de las actividades más indicadas para saber defender ideas que no necesariamente coinciden con la propia. Para aplicarlo, se necesita que el profesor motive a su realización y que haya un clima de libertad, de diálogo y respeto en el aula (Rojas, 2003). Con la práctica continua los alumnos pueden reconocer sus sentimientos,

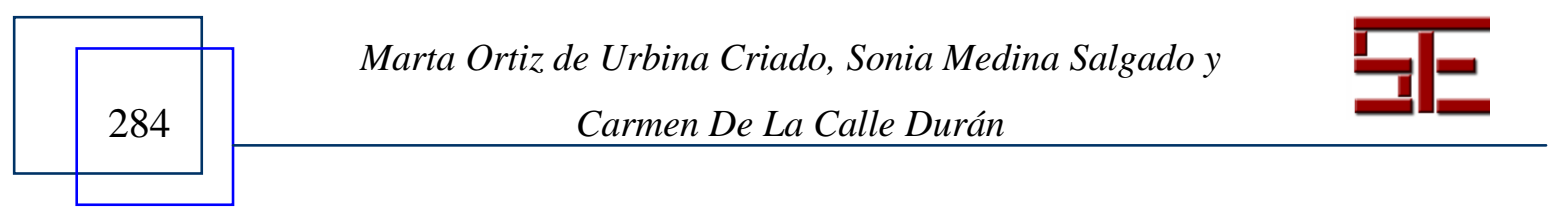




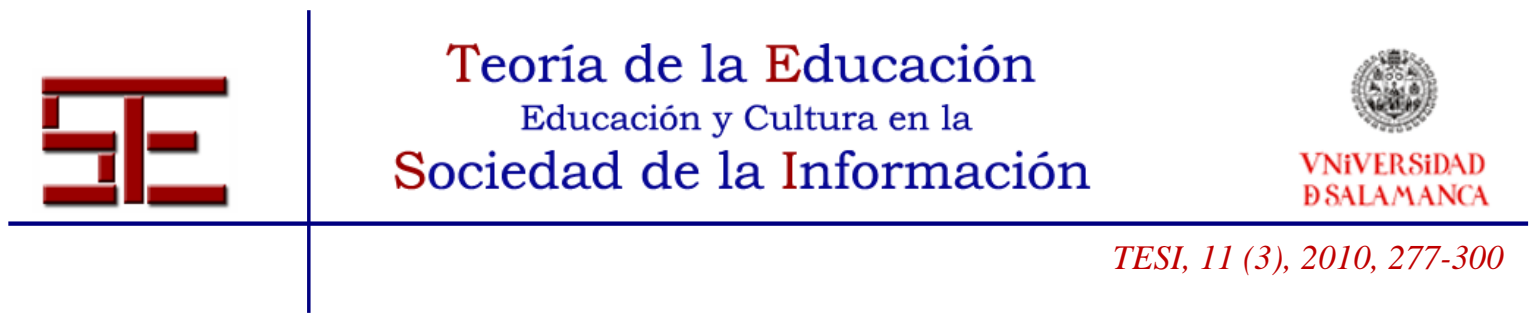

actitudes, valores, y entender sus conductas. Así mismo, permite aceptar a los demás, resolviendo conflictos y asumiendo con responsabilidad la toma de decisiones (Figura 1). Es, por tanto, una herramienta muy útil para el desarrollo de la docencia en el nuevo ámbito de la Educación Europea Superior basada en el acuerdo de Bolonia (1999).

\begin{tabular}{|c|c|}
\hline VENTAJAS & INCONVENIENTES \\
\hline $\begin{array}{c}\text { Permite desarrollar habilidades } \\
\text { interpersonales y de comunicación y } \\
\text { actitudes de aprendizaje (Ments, 1998; } \\
\text { Venable, 2001; Sogunro, 2004) }\end{array}$ & $\begin{array}{c}\text { Falta de aprendizaje por un feedback } \\
\text { inexperto por parte de los miembros de grupo } \\
\text { o del profesor }\end{array}$ \\
\hline $\begin{array}{l}\text { Aporta sentido de la realidad animando } \\
\text { a los estudiantes a investigar y } \\
\text { desarrollar situaciones de trabajo sin } \\
\text { riesgos (Rabinowitz, 1997; Jackson y } \\
\text { Walters, 2000; Venable, 2001) }\end{array}$ & $\begin{array}{l}\text { Los grandes grupos pueden salirse del } \\
\text { control del formador y que éste no pueda } \\
\text { monitorizar y proporcionar feedback } \\
\text { constantemente }\end{array}$ \\
\hline $\begin{array}{c}\text { Permite la resolución de conflictos } \\
\text { (Davis-Pack, 1989; Jackson y Walters, } \\
\text { 2000; Venable, 2001) }\end{array}$ & $\begin{array}{l}\text { Disfunción de papeles, conflicto y } \\
\text { ambigüedad de roles (Hughes et al., 1993) }\end{array}$ \\
\hline $\begin{array}{c}\text { Reduce la ansiedad y estimula la } \\
\text { asunción de riesgo ( Waters et al, } \\
\text { 1992; Venable, 2001) }\end{array}$ & $\begin{array}{c}\text { No se conocen totalmente la variedad de } \\
\text { roles y modos de usarlos de una manera } \\
\text { efectiva (Baruch, 2006) }\end{array}$ \\
\hline $\begin{array}{l}\text { Permite motivar a los estudiantes en } \\
\text { clase (Baruch, 2006) }\end{array}$ & $\begin{array}{l}\text { Se puede generar cierta disconformidad y } \\
\text { ansiedad entre los alumnos que hagan } \\
\text { fracasar la técnica }\end{array}$ \\
\hline \multirow{2}{*}{$\begin{array}{l}\text { Acelera el aprendizaje (Sogunro, } \\
\text { 2004): permite a los estudiantes } \\
\text { explorar roles de gestores y } \\
\text { especialistas y dinámicas de } \\
\text { comunicación en pequeños grupos } \\
\text { (Jackson y Walters, 2000) }\end{array}$} & $\begin{array}{c}\text { Si las situaciones son muy complejas y } \\
\text { requieren conocimiento especializado (otros } \\
\text { especialistas) pueden no ser exitosas (Ments, } \\
\text { 1998; Sogunro, 2004) }\end{array}$ \\
\hline & \begin{tabular}{|c|} 
Falta de compromiso, tiempo de preparación \\
o inexperiencia del profesor/formador para \\
realimentar a los alumnos (Sogunro, 2004)
\end{tabular} \\
\hline
\end{tabular}

Figura 1. Resumen de las ventajas e inconvenientes del juego de rol

En el libro blanco de la Agencia Nacional de Evaluación de la Calidad y Acreditación (ANECA, 2005) se recogen las competencias necesarias para la obtención de los diferentes títulos de grado. Para las titulaciones relacionadas con la empresa se hace

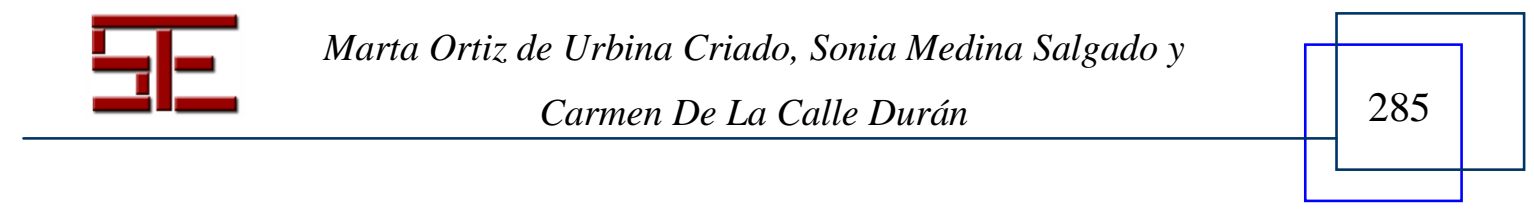




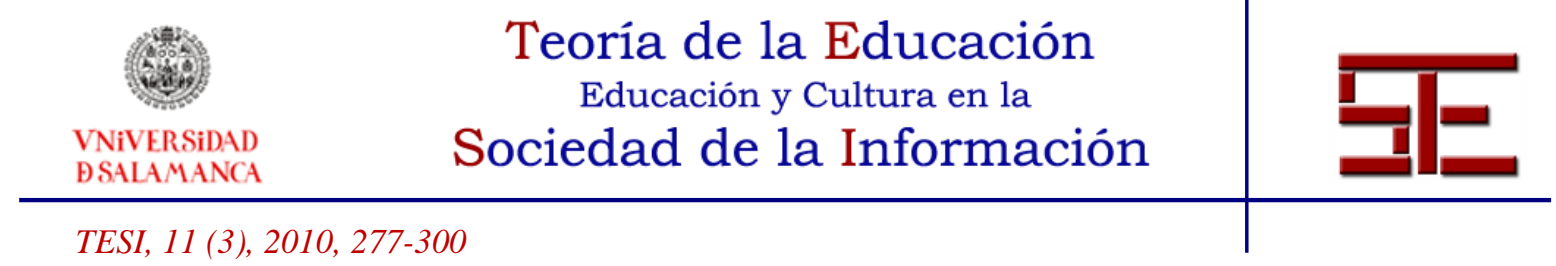

especial hincapié no sólo en los conocimientos sino en las habilidades o competencias que son necesarios para entender el mundo empresarial. Las competencias se clasifican en: específicas, genéricas y transferibles. Las competencias específicas incluyen los conocimientos técnicos, socioeconómicos (entorno en el que se desenvuelven las empresas) y de soporte. Son los conocimientos básicos y los conocimientos complementarios. Las competencias genéricas se refieren a las capacidades organizativas y de relación con el entorno y pueden ser instrumentales, personales o sistémicas. De entre las ocho competencias instrumentales, el juego de rol permite desarrollar cuatro de ellas: la capacidad de análisis y síntesis, la capacidad de organización y planificación, la capacidad para la resolución de problemas y la capacidad para tomar decisiones. En el caso de las competencias personales, el juego de rol también permite desarrollar cuatro de ellas: capacidad para trabajar en equipo, habilidad en las relaciones personales, capacidad crítica y autocrítica y trabajar en entornos de presión. En cuanto a las competencias sistémicas de las siete existentes se destacarían dos: la capacidad de adaptación a nuevas situaciones y el liderazgo. Finalmente, las competencias transferibles son las que ayudan a cerrar la brecha entre teoría y mercado laboral. Entre las cuatro existentes, el juego de rol puede ser útil para desarrollar la capacidad de aplicar los conocimientos en la práctica.

Según el libro blanco, el Graduado en Empresa o en titulaciones relacionadas debe saber identificar y anticipar oportunidades, asignar recursos, organizar la información, seleccionar y motivar a las personas, tomar decisiones, alcanzar objetivos propuestos y evaluar resultados. Para ello, debe haber adquirido el carácter de un experto, una persona práctica, con habilidades claras, experimentada en su campo, que pueda abordar problemas de gestión con criterios profesionales y con el manejo de instrumentos técnicos. A través de la experiencia docente, hemos observado que la clase magistral no cubre este tipo de habilidades. El alumno tiene que aprender a "saber hacer" y a enfrentarse a situaciones que no siempre le son conocidas. Cuando se desarrolla un juego de rol es con la idea de la resolución de un problema que a priori le es conocido pero que variará en la medida en la que se tiene que interactuar con otros agentes (compañeros), con su propia opinión y forma de ser y actuar.

Por lo tanto, esta técnica, en el ámbito de la formación, se considera como una herramienta efectiva y poderosa para situaciones de aprendizaje variadas en múltiples disciplinas del conocimiento (Figura 2): el estudio de sistemas complejos, la exploración de distintos enfoques de un problema (Jackson y Walters, 2000; Baruch, 2006), el logro de pensamiento crítico (Venable, 2001). Además, y como señala Sogunro (2004) se considera como una herramienta útil para desarrollar los niveles más

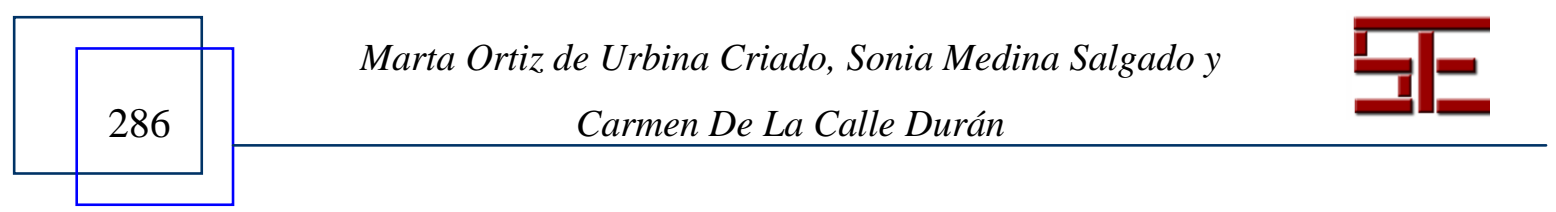




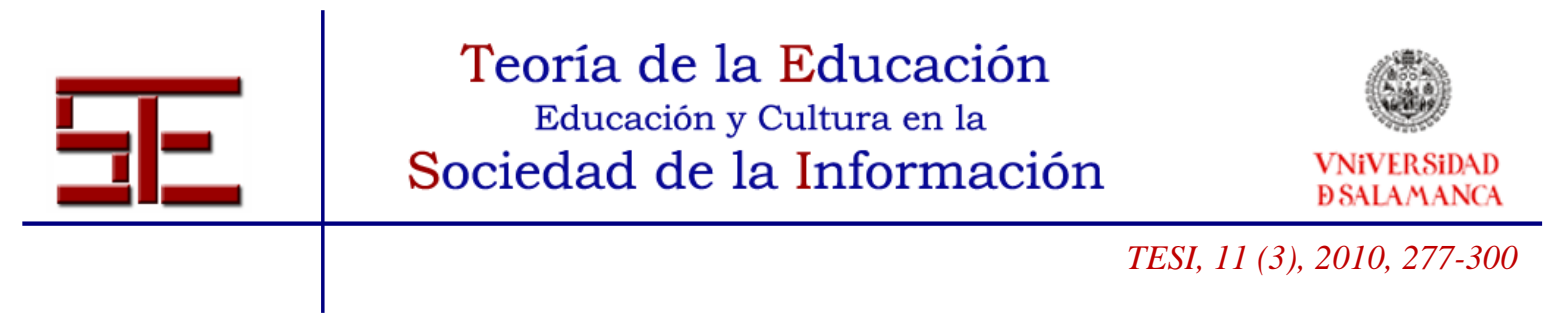

altos del dominio cognitivo del aprendizaje, tanto individual como en grupo, como, por ejemplo: el análisis y la aplicación, la comprensión y el conocimiento. De hecho es posible encontrar experiencias muy concretas como la de Begley (1998) que muestra un ejemplo de juego de rol para enseñar a sus alumnos a manejar situaciones reales de reestructuración en una empresa. También podemos destacar la experiencia de Brown (1994) que utiliza el juego de rol para introducir comportamientos éticos en la enseñanza, en concreto, en el caso de la gestión financiera.

\begin{tabular}{|c|}
\hline EJEMPLOS \\
\hline Estética (Venable, 2001) \\
\hline $\begin{array}{c}\text { Formación en liderazgo, management, administración, supervisión, enseñanza, } \\
\text { trabajo social, relaciones públicas, enfermería, etc. (Sogunro, 2004) }\end{array}$ \\
\hline $\begin{array}{c}\text { Capacitación para la gestión y para la enseñanza de ciencias naturales, política, } \\
\text { psicología, economía, en concreto, química analítica (Jackson y Walters, 2000) }\end{array}$ \\
\hline $\begin{array}{c}\text { Habilidades de comunicación para sistemas de información profesionales (Chen; } \\
\text { Muthitacharoen y Frolick, 2003) }\end{array}$ \\
\hline Dirección de operaciones (Yazici, 2004) \\
\hline Principios de marketing (Muñoz y Huser, 2008) \\
\hline Aprendizaje de idiomas (Inda et al., 2008) \\
\hline Praxis jurídica (Garrido y Romero, 2009) \\
\hline Educación familiar y modelos de orientación en psicopedagogía familiar (Lara y \\
Rivas, 2009)
\end{tabular}

Figura 2. Ejemplos de aplicaciones del juego de rol

Dados los antecedentes expuestos, parece claro que una herramienta de aprendizaje colaborativo y experiencial como el juego de rol debería ser usada en la formación universitaria en la mayor parte de las disciplinas del conocimiento si se pretende formar expertos competentes y bien capacitados para el ejercicio profesional real en el entorno actual. Sin embargo, la realidad nos demuestra que pocos educadores la incorporan regularmente en su estructura pedagógica básica relegándola a pequeñas actividades en el aula (Jackson y Walters, 2000). De hecho, en el caso español, no ha sido hasta hace poco tiempo cuando se han empezado a publicar experiencias concretas de la aplicación del juego de rol en la enseñanza universitaria como un modo de innovación docente (por ejemplo, Inda et al., 2008; Garrido y Romero, 2009; Lara y Rivas, 2009).

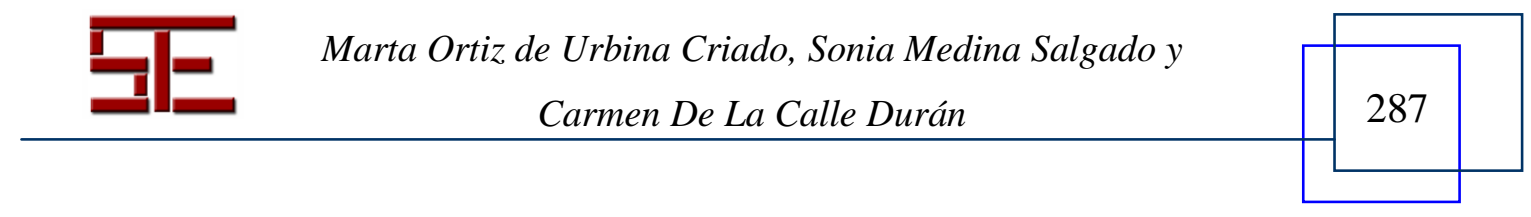




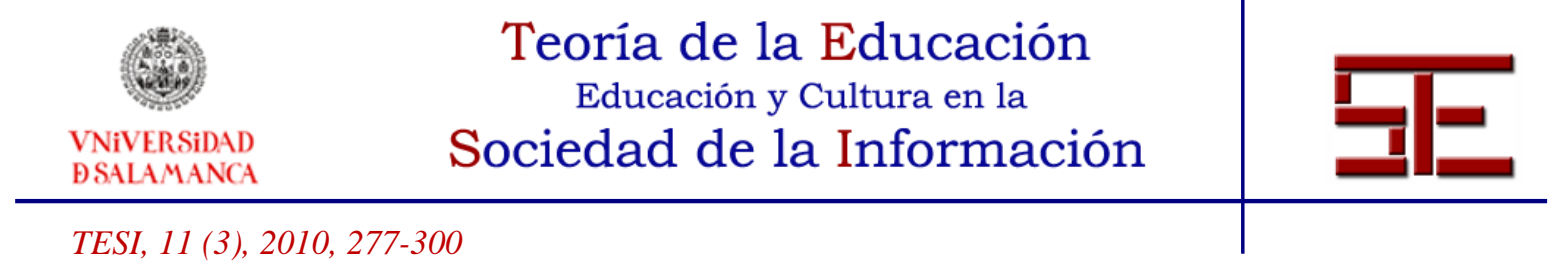

En este sentido, sería muy interesante tratar de investigar, con mayor profundidad, en qué situaciones de aprendizaje se ha aplicado esta técnica y cómo son los resultados que se han alcanzado. En el apartado siguiente se explicará el desarrollo y los resultados que se han obtenido en la aplicación de esta técnica en la asignatura de 'Dirección de Recursos Humanos'.

\section{3.- UNA APLICACIÓN PRÁCTICA DEL JUEGO DE ROL}

En el siglo veintiuno marcado por la era de las nuevas tecnologías y globalización de los mercados, nadie cuestiona ya la importancia de una adecuada gestión o dirección de los recursos humanos para el éxito e incluso la supervivencia de las empresas, máxime cuando es el sector servicios el de mayor importancia mundial. Sin embargo, si desde un punto de vista de eficiencia los recursos se gestionan para minimizar sus costes, gestionar recursos humanos va más allá. La dificultad de su gestión radica precisamente en su carácter humano. El hombre es un ser complejo, cada uno con sus propias inquietudes y deseos. Por tanto, dirigir los recursos humanos es una tarea básica y compleja para las empresas de hoy. Aunque la Dirección de Recursos Humanos debería ser individualizada y personalizada, desde un punto de vista de coste-eficiencia esto no es posible, los directivos deben ser conscientes de las peculiaridades de su personal para saber extraer lo mejor de cada uno de ellos.

Con esta nueva visión de la actual Dirección de Recursos Humanos, nos planteamos hacer un juego de rol para un proceso de reclutamiento. Éste es uno de los principales desafíos a los que se tienen que enfrentar las empresas y, en concreto, el departamento de recursos humanos. El reclutamiento supone la búsqueda y selección del personal para ocupar los puestos de trabajo previamente diseñados en el análisis y planificación de los mismos (De La Calle y Ortiz-de-Urbina, 2004). No es tarea fácil encontrar candidatos cualificados y seleccionar al candidato que mejor se adapte a las características del puesto de trabajo. Aunque existen herramientas y prácticas que facilitan el proceso de reclutamiento y selección, el papel del encargado del mismo es muy importante. Por eso consideramos que a través del juego del rol los alumnos pueden interiorizar la importancia del papel del candidato y del futuro directivo-seleccionador.

\section{1.- Descripción de la práctica realizada en el aula}

Nuestra experiencia en el uso del juego de rol proviene de su aplicación en la asignatura 'Dirección de Recursos Humanos' impartida en varias titulaciones de la Universidad

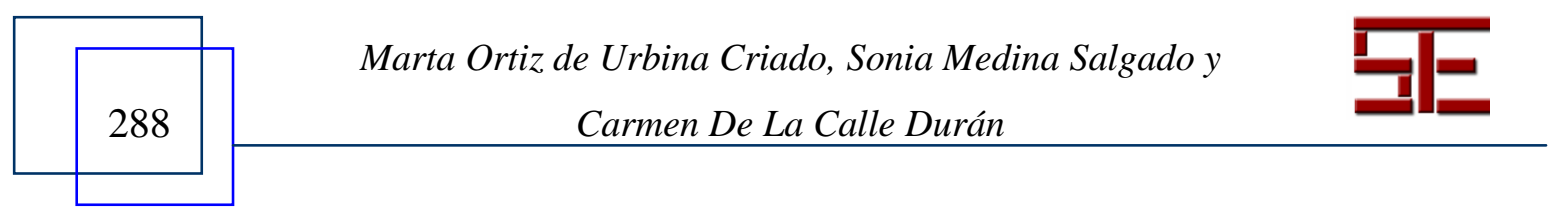




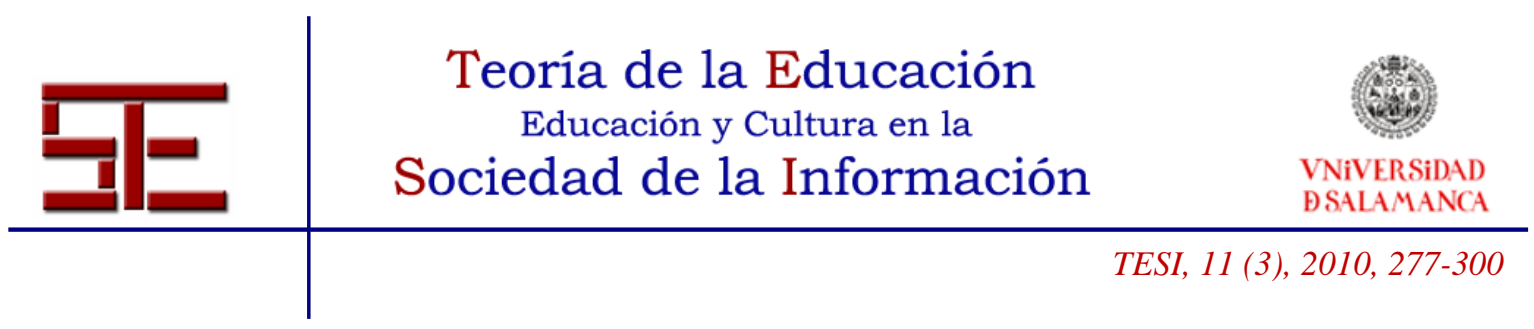

Rey Juan Carlos: Licenciatura en Administración y Dirección de Empresas (asignatura optativa), Diplomatura en Turismo (asignatura troncal), Master Oficial en Relaciones Laborales (asignatura obligatoria). Esta asignatura, perteneciente al área de Organización de Empresas, tiene como objetivo el análisis de los Recursos Humanos (RR.HH.) desde la perspectiva de gestión y dirección estratégica, considerando que éstos son el principal recurso de la empresa. Sin embargo, resulta interesante para cualquier titulación y por eso se está incluyendo en muchos de los nuevos grados, no sólo en los de empresa.

En el ámbito de la dirección de los Recursos Humanos, una de las prácticas más importantes es el proceso de reclutamiento y selección, que se caracteriza por una serie de etapas, que van desde la búsqueda de candidatos, la preselección, las pruebas de selección hasta la contratación del candidato seleccionado. Por eso, una de las prácticas propuestas en la asignatura de Dirección de RR.HH. es un juego de rol sobre un proceso completo de contratación de personal en una empresa. Para incluir todas las etapas del proceso de contratación, se planteó la actividad en varias fases o etapas que se fueron desarrollando en distintas sesiones/clases y a lo largo de varias semanas. Los grupos en los que se aplicó esta técnica tenían una media de 30 alumnos y las sesiones duraban dos horas.

La puesta en marcha de esta actividad requiere la elaboración por parte del profesor de un material y de una guía de la actividad en la que se explica la metodología que se va a seguir, el contenido de cada fase y los recursos que se van a utilizar. En la sesión de información, el profesor explica a los alumnos qué es el juego de rol, cuál es su utilidad y sus objetivos y cómo se estructura. A continuación, el profesor entrega a los alumnos la guía de la actividad en la que se incluye la oferta de trabajo que se va a tomar como referencia, es decir, el puesto de trabajo para el que se desea seleccionar a un candidato, y asigna a cada grupo de alumnos o alumno (según el número de alumnos que haya en la clase) uno de los papeles o roles. Los roles que hay en esta actividad son los siguientes: candidatos al puesto, pre-seleccionadores, encargados de las pruebas psicotécnicas, entrevistadores, encargados de la tramitación del contrato y encargados de la elaboración de un plan de formación y carrera para el candidato elegido al final del proceso. Por ejemplo, para un grupo de 30, se asignaba el papel de candidato a 7 alumnos, se creaban 4 grupos de tres personas y un grupo de 1 para la parte de tramitación del contrato. Cada grupo o alumno tiene que preparar su papel para poder desempeñarlo el día que se represente en clase. A continuación vamos a comentar cada una de las etapas que se desarrolla para la realización de la práctica.

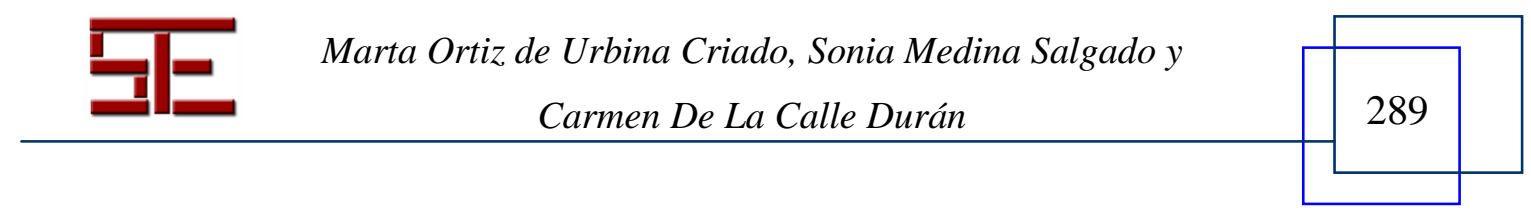




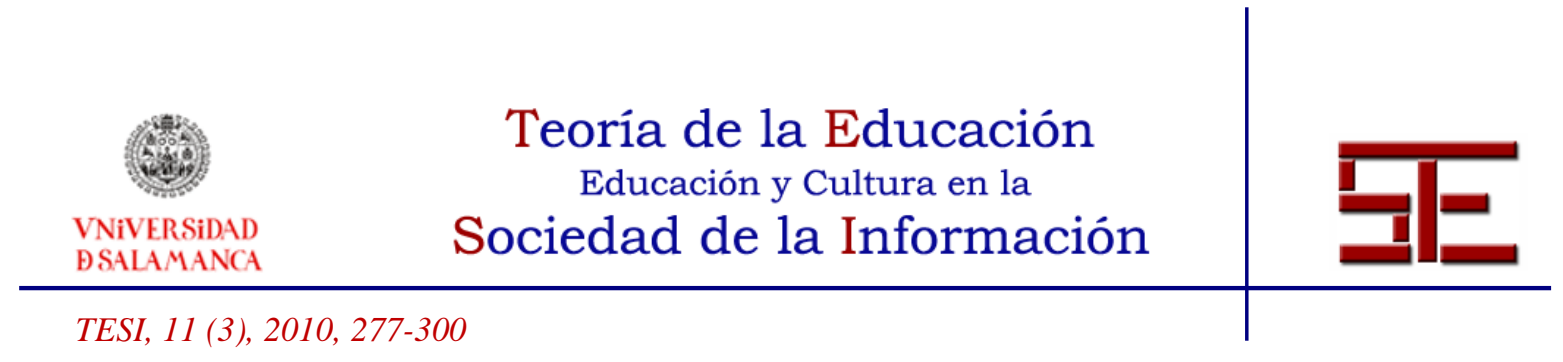

Etapa 1: reclutamiento y preselección

Para entender el proceso de reclutamiento y preselección de las empresas es necesario conocer qué tienen que hacer los candidatos y qué suelen hacer las empresas. Por eso, en esta etapa se pretende, por una parte, enseñar al alumno cómo se elabora un Currículum Vitae (CV) y una carta de presentación, adaptados ambos a la oferta de trabajo y, por otra, mostrar cómo se suelen hacer los procesos de preselección en las empresas. La información necesaria tanto para la elaboración del CV y la carta de presentación como para el proceso de preselección se ha obtenido fundamentalmente de Internet. En la práctica realizada en el aula, el grupo de alumnos encargado de hacer el CV y la carta de presentación, previamente, había realizado una labor de búsqueda y de análisis de esta información. En la sesión 1, cada alumno de este grupo había preparado su CV y su carta de presentación y en el aula explicaba a sus compañeros cómo los había elaborado. Por otra parte, en esta misma sesión, el grupo encargado de la preselección se había encargado de elaborar, a partir de la oferta de trabajo, una descripción del puesto y el perfil profesional y de buscar información sobre los criterios a aplicar para hacer la preselección de CV. En el aula, este grupo realizaba una simulación de la selección de los CV, explicando a sus compañeros en qué consistía el proceso. El resultado de esta primera fase fue la selección de la mitad de los CV entregados. Estos candidatos pasaban a la etapa siguiente.

\section{Etapa 2: pruebas psicotécnicas y técnicas}

Una vez que la empresa ha realizado el filtrado de los CV recibidos para la oferta de trabajo (preselección), realizará una o varias pruebas a los candidatos: pruebas psicotécnicas y técnicas, entrevistas personales y/o técnicas. En esta segunda sesión se pretende hacer una simulación de las pruebas psicotécnicas y técnicas. Para preparar la actividad, se utilizó información obtenida de Internet y algunos manuales sobre este tipo de pruebas. El grupo de alumnos a los que se les ha asignado este papel, asesorados por el profesor, preparaban antes de la clase las pruebas, las soluciones y su interpretación y en el aula se aplicaban estas pruebas a los candidatos que habían superado la preselección. Tras realizar las pruebas, el equipo encargado de esta actividad, comentaba los resultados obtenidos y la interpretación de éstos para cada candidato. Al finalizar esta sesión, el objetivo era quedarse sólo con tres candidatos, que son los que pasarían a la fase siguiente.

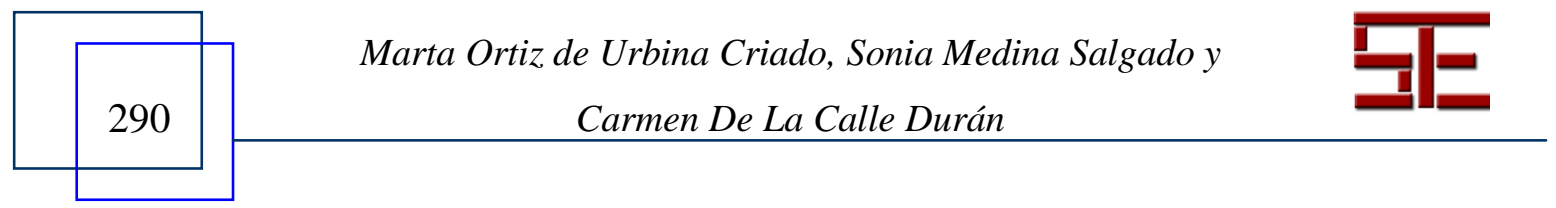




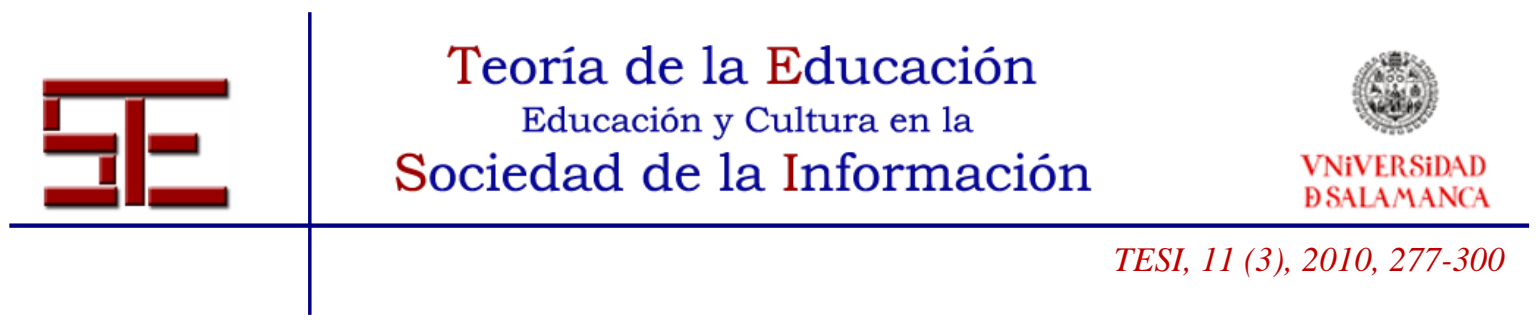

\section{Etapa 3: entrevistas personales}

En esta fase se pretende mostrar a los alumnos cómo se desarrollan las entrevistas personales de selección y ofrecerles tanto el punto de vista de las empresas como el de los candidatos. Para preparar la actividad se utilizaron tanto manuales sobre entrevistas personales de selección como información obtenida de Internet. Para la realización de esta etapa, los candidatos que había superado la fase dos tenían que buscar información sobre cómo prepararse para una entrevista de trabajo y sobre la empresa que hacía la oferta de trabajo. Por otra parte, el grupo de entrevistadores tuvo que buscar información sobre cómo se hacía una entrevista personal y prepararla. En el aula, se realizaban las tres entrevistas personales. Durante el desarrollo de cada entrevista, el resto de la clase, asesorada por los entrevistadores, tenía que tomar nota del comportamiento y de las respuestas de los entrevistados. Una vez realizadas las entrevistas, el grupo de entrevistadores se reunía fuera de la clase para dar una calificación y hacer una evaluación de cada candidato y, a su vez, se realizaba un debate con toda el aula para conocer su opinión. Una vez realizadas las evaluaciones, el grupo de entrevistadores explicaba a la clase, de forma justificada, las calificaciones que había dado a cada candidato y se abría un debate para llegar a un acuerdo sobre la calificación final. Los candidatos no estaban presentes en la parte de evaluación debido a que todavía tenían que someterse a una entrevista de grupo y no debían saber en qué posición se encontraban.

\section{Etapa 4: entrevistas de grupo y selección del candidato}

En esta fase se pretende mostrar a los alumnos cómo se desarrollan las entrevistas de grupo en los procesos de selección. Para preparar la actividad, se utilizaron principalmente manuales sobre entrevistas de grupo. Al igual que en la etapa anterior, antes de la clase, los candidatos tenían que buscar información sobre cómo prepararse para una entrevista de grupo y el grupo de entrevistadores tuvo que buscar información sobre cómo se hacía este tipo de entrevistas y prepararla. En el aula, se realizó la entrevista de grupo. Durante el desarrollo de la entrevista, el resto de la clase, asesorada por los entrevistadores, tenía que tomar nota del comportamiento y de las respuestas de los entrevistados. Finalizadas las entrevistas, el grupo de entrevistadores se reunía fuera de la clase para dar una calificación y hacer una evaluación de cada candidato. Mientras el resto de la clase debatía su opinión. Una vez realizadas las evaluaciones, se iniciaba un proceso similar al de la etapa 3. Los candidatos en esta ocasión sí que estaban presentes $y$, al final del debate, el grupo de entrevistadores explicaba a los candidatos la

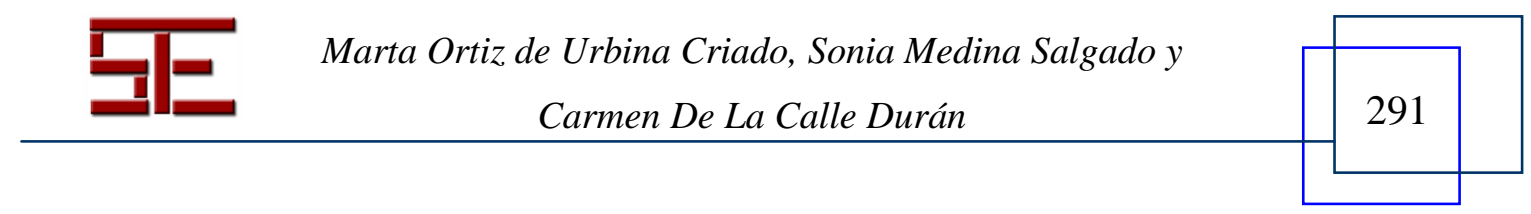




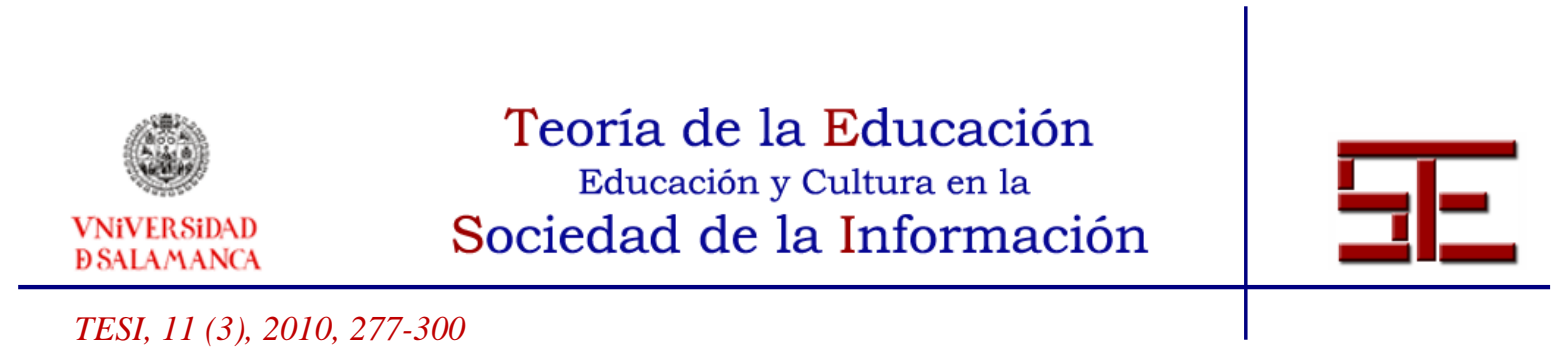

calificación que habían obtenido en las dos entrevistas y, por lo tanto, quién era el candidato elegido de los tres.

Etapa 5: contratación, plan de formación y plan de carrera

Una vez seleccionado el candidato, se pretendía mostrar a los alumnos cómo es un contrato de trabajo y cómo se elabora un plan de formación y de carrera para un trabajador. La información necesaria para elaborar esta parte de la actividad se obtuvo tanto de manuales como de Internet. En esta sesión un alumno se encargaba de elaborar el contrato y otro grupo de alumnos se encargaba de preparar el plan de formación y de carrera teniendo en cuenta las características de la empresa y el perfil del puesto y del candidato. En la clase se simulaba una reunión con el candidato seleccionado en la que se le comentaban las condiciones del puesto de trabajo y el contrato. Si el candidato aceptaba el puesto se le explicaba el plan de formación y de carrera. En esta sesión, al finalizar la actividad, se iniciaba un debate para que los alumnos opinaran sobre la práctica realizada, comentasen sus ventajas y sus inconvenientes y propusieran formas de mejorar la metodología y el desarrollo de la práctica.

\section{2.- Resultados y discusión}

En todas las ocasiones, al finalizar la práctica, el profesor y los alumnos analizaban el desarrollo de la actividad, las dificultades y su resolución y la utilidad de la técnica para el aprendizaje.

La mayoría de los grupos (95\%) consideraba que la actividad se había desarrollado según lo planificado y que todos los alumnos se habían comprometido en mayor o menor medida con la actividad. En una de la clases, en cambio, hubo problemas para realizar todas las fases de la práctica porque algunos de los alumnos no hicieron su parte del trabajo, a pesar de que el profesor al inicio de la práctica advirtió a los alumnos que si uno sólo de ellos falla (no se preparaba el papel, no iba a clase el día que hay que hacer la actividad, etc.), la actividad no se podría hacer y repercutiría en la nota de toda la clase. En este caso el profesor optó por continuar con la actividad y penalizar sólo a los alumnos que habían fallado. Para poder continuar con la actividad, otros alumnos que hacían un papel similar o el profesor, según las circunstancias, asumieron el papel del alumno que falló.

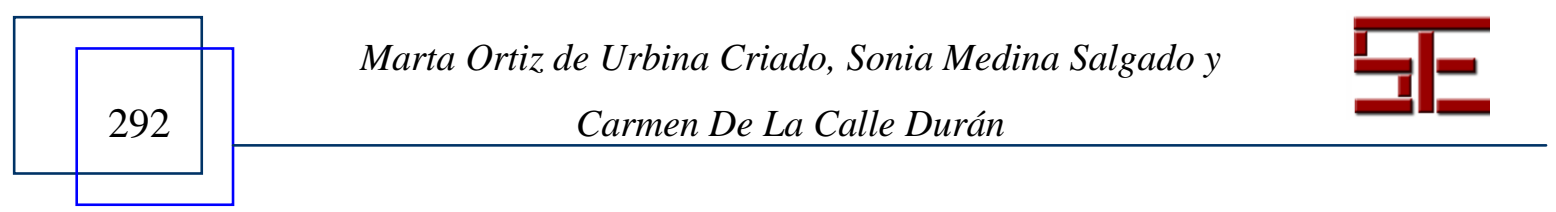




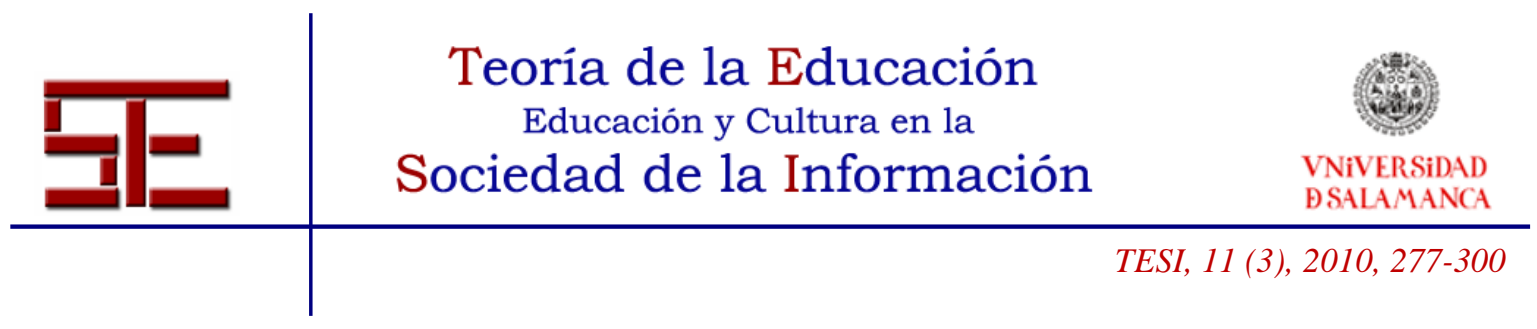

Otra de las cuestiones que se analizaba al final de la práctica eran las dificultades para su preparación y desarrollo. Prácticamente la totalidad de los alumnos (97\%) consideraban que lo más difícil era la preparación del rol o de la parte de la actividad que les correspondía, es decir, la búsqueda de la información necesaria y, sobre todo, el análisis de la misma. Otro de los problemas que encontraban era que tenían que trabajar en grupo y muchos tenían problemas para reunirse cara a cara. Sin embargo, al final de la actividad, muchos grupos comentaron que habían buscado la forma de trabajar en grupo sin necesidad de verse cara a cara en todas las ocasiones. Algunos grupos había usado el correo electrónico para estar en contacto y, en alguna ocasión, se habían reunido para concretar cosas o cerrar el trabajo. Otros grupos había utilizado la plataforma BSCW (Basic Support for Cooperative Work), que es una aplicación informática que permite el trabajo en grupo de manera sencilla a través de Internet: permite almacenar y organizar información, compartirla con otras personas, realizar debates, disponer de agendas conjuntas, etc. Finalmente, otro de los problemas que se encontraron era que alguno de los miembros del grupo no hacía su trabajo y eran los demás los que tenían que hacerlo.

La opinión general de los alumnos que han participado en esta práctica es que el juego de rol es una actividad divertida que enseña de forma práctica no sólo el contenido de la actividad sino la importancia del trabajo en grupo, de que todos los miembros del grupo se responsabilicen y aporten su parte. Así mismo, consideraban que era una forma de acercarse a la realidad empresarial y de estar mejor preparados para el mundo laboral.

Desde el punto de vista del profesor, la experiencia ha sido muy positiva y enriquecedora, ya que con esta actividad se produce una retroalimentación entre alumno y profesor: el profesor además de enseñar de forma práctica y divertida una parte de la asignatura aprende de los alumnos. En general, la práctica ha funcionado muy bien, la mayoría de los alumnos ha trabajado en grupo y ha participado activamente.

Una de las cosas que hemos observado al realizar esta actividad es que la motivación del alumno suele ser muy alta y que los propios alumnos son los que tratan de motivar a los otros compañeros para que la actividad salga bien. Siempre surge algún conflicto porque algún alumno no tiene la intención de participar o porque no hay acuerdo en cuanto a la forma de preparar la actividad o porque unos perciben que hacen más trabajo que los otros, pero, en general, la clase es capaz de buscar soluciones para evitar que la actividad se quede sin hacer. En este caso, el profesor tiene que hacer de mediador y potenciar los comportamientos cooperativos premiándolos, tanto con nota como con

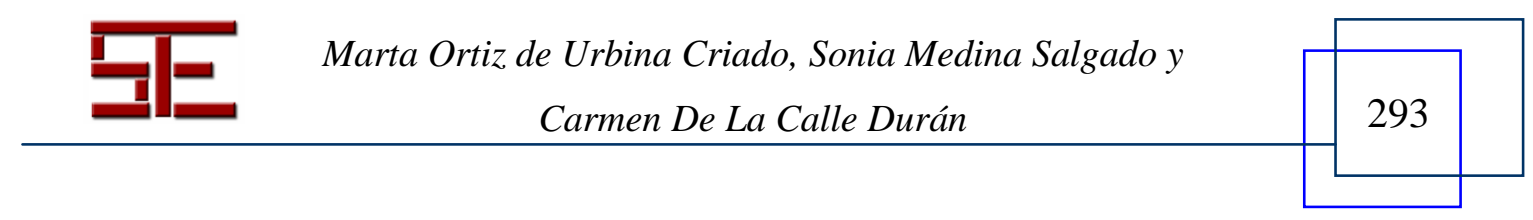




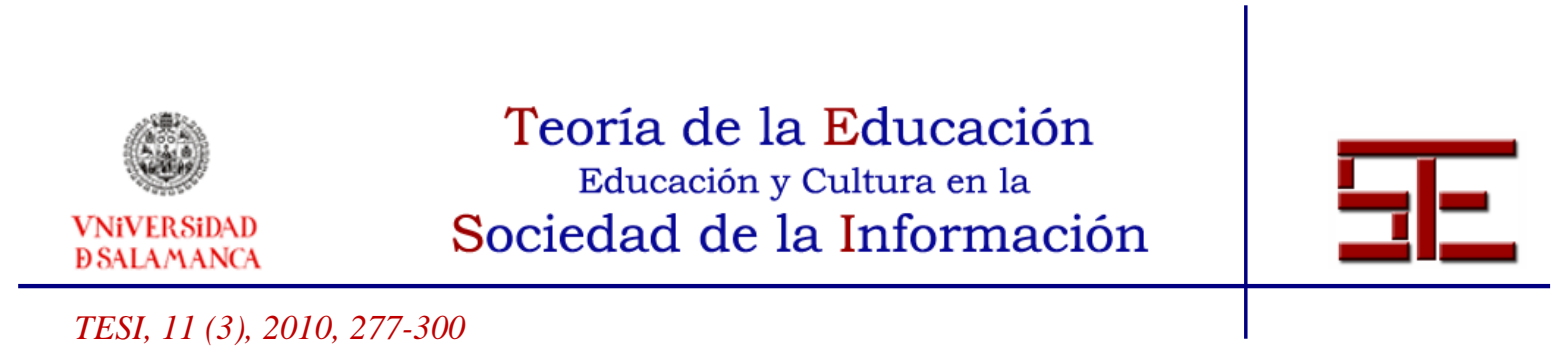

reconocimiento explícito frente a la clase, y penalizar los comportamientos individualistas o de rechazo a los demás. Por eso, creemos que tan importante es la preparación de la actividad como su sistema de evaluación ya que éste sirve de elemento motivador. Para que estas actividades tengan efecto y potencien la capacidad de aprendizaje cooperativo, el sistema de evaluación tiene que premiar los comportamientos cooperativos. En este caso, se decidió que una parte de la nota final de la asignatura se obtuviese por el resultado final de la práctica (la misma para toda la clase) y otra parte de la nota se obtuviera por la aportación individual de cada alumno a la actividad. Nuestra opinión es que tanto la transmisión de conocimientos como el aprendizaje del alumno y la evaluación tienen que tener una parte individual y una colectiva.

En relación con el sistema de evaluación, Inda et al. (2008) exponen una interesante forma de evaluación de un juego de rol utilizado para el aprendizaje de la lengua francesa y llegan a la conclusión de que los estudiantes con mejores resultados eran los que habían seguido la enseñanza de la lengua francesa mediante este juego. Según defiende Boud (1998) el proceso de evaluación es imprescindible en el proceso de aprendizaje: "Los métodos y requisitos de la evaluación probablemente tienen más influencia en cómo y qué aprenden los estudiantes, que cualquier otro factor individual. Esta influencia es posible que tenga mayor importancia que el impacto de los materiales de la enseñanza”. Inda el al. (2008) aplican además de la evaluación clásica, que consiste en la valoración de la actividad del estudiante por parte del profesor (heteroevaluación), otras prácticas evaluativas como la evaluación del estudiante de su propio rendimiento individual (autoevaluación), la cual permite desarrollar su capacidad crítica y favorecer su independencia y creatividad; la evaluación de un grupo de estudiantes de su rendimiento como grupo (coevaluación), que favorece la negociación entre los distintos miembros del grupo; y la evaluación del alumno por parte de sus compañeros (heteroevaluación). En nuestro caso, para la evaluación de la práctica realizada en el aula se aplicó la heteroevaluación, ya que el profesor fue el que asignó a cada alumno una nota. Sin embargo, en el aula el profesor propuso a la clase que evaluaran el papel desempeñado por sus compañeros y, al final de cada sesión, el profesor preguntaba a la clase su opinión sobre la preparación de la práctica realizada ese día en el aula y su puesta en escena. También al final de la actividad, el profesor abrió un debate para que los alumnos se autoevaluasen y evaluasen a sus compañeros de clase y, en su caso, a sus compañeros de grupo. Siguiendo la propuesta de Inda et al. (2008), pensamos que en próximas aplicaciones de la práctica sería interesante que la

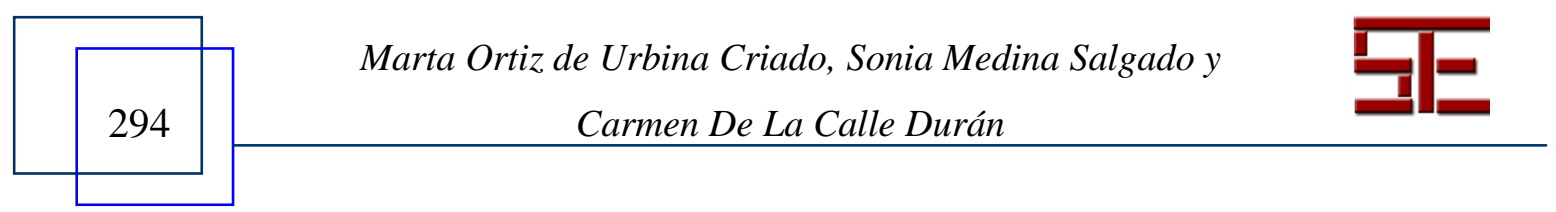




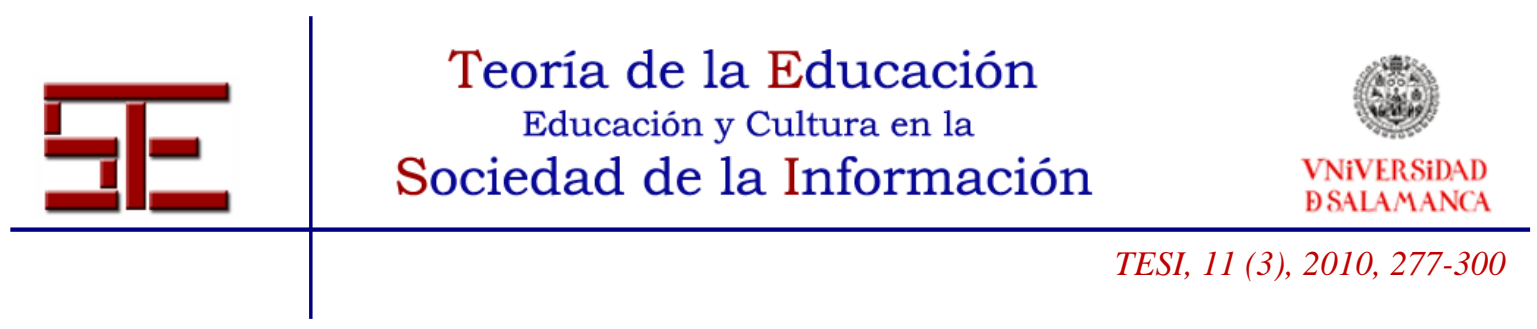

nota del alumno esté compuesta por las notas obtenidas a partir de los cuatro tipos de evaluaciones.

\section{4.- CONCLUSIONES}

Los juegos han constituido una poderosa herramienta de aprendizaje de conductas y actitudes necesarias para el eficiente desempeño sociocultural. El juego es una actividad fundamental para el desarrollo humano. Comúnmente se juega para divertirse, para entretenerse; sin embargo, hay quien afirma que se juega principalmente para aprender, aunque ésta sea una intención inconsciente (Crawford, 1982). Otros consideran al juego como una actividad voluntaria, primordialmente social, en la que se está en relación con otros y se aprenden pautas de comportamiento, valores y la cultura (Gros, 2000). Por su parte, Cañeque (1991) considera que el juego educativo tiene importantes funciones. Entre otras destacan: ser un medio fundamental para la estructuración del lenguaje y el pensamiento, producir una sensación continua de exploración y descubrimiento, reducir la sensación de gravedad frente a errores y fracasos, implicar cierta participación activa del jugador y desarrollar la creatividad. Precisamente es esta última función la que Trigo (2006) destaca especialmente, considerando que el juego, como creatividad lúdica, es el modelo que conduce a los progresos tecnológicos y a los cambios sociales. En definitiva, se puede afirmar que jugar constituye una estrategia fundamental para estimular el desarrollo integral de las personas en general (Marcano, 2008, 96).

El objetivo de este trabajo ha sido demostrar el efecto y la utilidad del juego de rol para la enseñanza en el área de Organización de Empresas. En concreto, se ha analizado y explicado la puesta en práctica de esta herramienta en el aula para la asignatura de 'Dirección de Recursos Humanos' impartida en varias titulaciones durante varios cursos académicos.

La actual visión de la gestión de los Recursos Humanos se plantea como una forma sistémica que consta de diferentes procesos; entre ellos, los procesos de reclutamiento y selección se consideran fundamentales y van desde la búsqueda, preselección y pruebas de selección de candidatos, hasta la contratación del candidato seleccionado. Por eso, una de las prácticas propuesta en las asignaturas de 'Dirección de RRHH' es un juego de rol sobre un proceso completo de contratación del personal en una empresa. Para poder acaparar todo el proceso contratación, la práctica se dividió en varias fases que se fueron desarrollando en distintas sesiones/clases a lo largo de varias semanas.

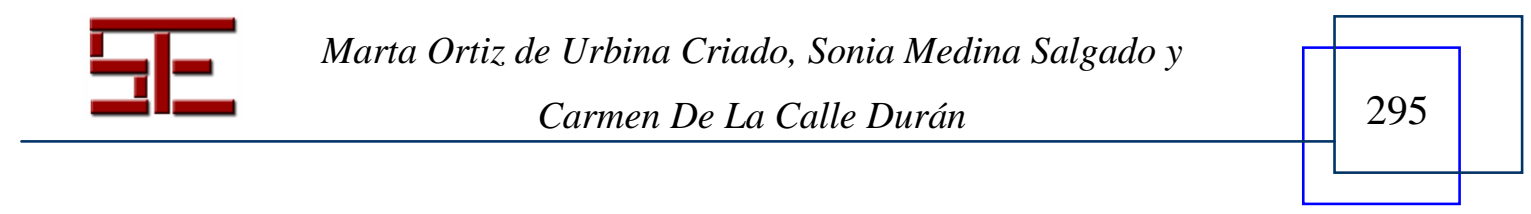




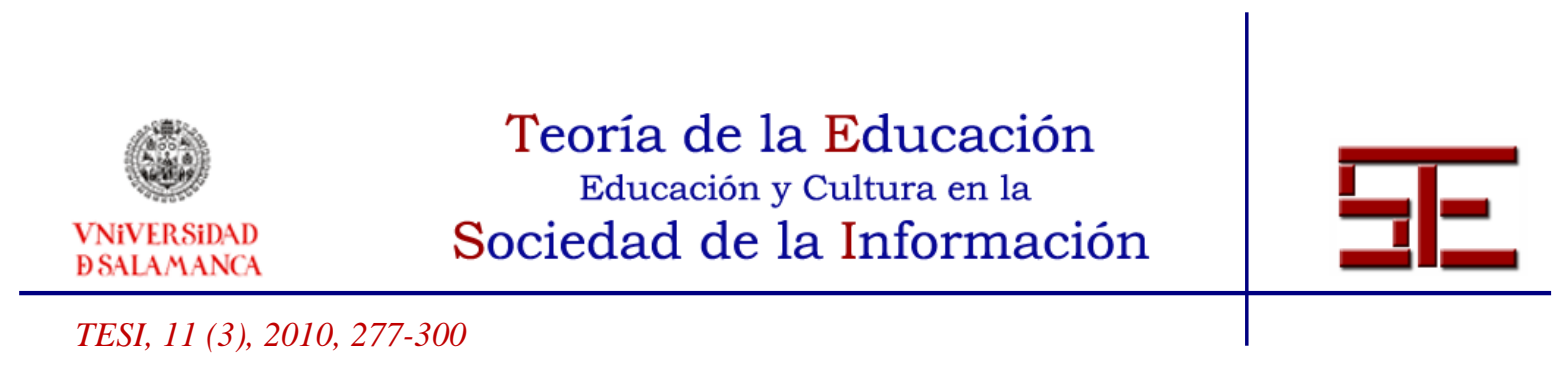

La experiencia directa nos ha mostrado que con esta actividad se logra que alumno aprenda e interiorice el proceso de contratación de personal en las empresas y que perciba mejor la realidad empresarial enfrentándose a estas situaciones, tanto desde el punto de vista del empleador como del posible candidato. Además, la aplicación del juego de rol en el aula ha facilitado el aprendizaje colaborativo al tener en cuenta los cinco componentes esenciales para su buen funcionamiento (Padilla et al., 2008): interdependencia positiva, exigibilidad personal, interacción positiva cara a cara, habilidades interpersonales y de grupo, y autoanálisis del grupo.

Tras el análisis del juego de rol realizado en el aula se pone de manifiesto la utilidad de esta técnica para trabajar la empatía y la comprensión con los demás. Tanto los alumnos como los profesores perciben las dificultades y la eficacia de esta herramienta para el aprendizaje de los niveles más altos del dominio cognitivo. El juego de rol permite aceptar a los demás resolviendo conflictos y asumiendo con responsabilidad la toma de decisiones. Al haber más sentido de la realidad y mayor implicación emocional, el alumno puede reconocer los sentimientos, actitudes y valores que caracterizan, en algunos casos, su conducta sin asumir grandes riesgos. Ello le capacita para resolver y poner en práctica nuevas formas de resolver problemas en el futuro.

En conclusión, en este trabajo hemos mostrado de forma práctica la utilidad del juego de rol como herramienta para el aprendizaje y desarrollo de competencias como la cooperación en el trabajo, la resolución de conflictos, la toma de decisiones, la resolución de problemas y la gestión de sistemas complejos. La relevancia del uso de la técnica en el contexto de innovación docente actual asociado al EEES, que conlleva un cambio de filosofía de la enseñanza en el ámbito de la gestión empresarial, así como su escaso uso en el área de Organización de Empresas justifican el interés de este trabajo.

\section{5.- BIBLIOGRAFÍA}

Aymerich Andreu, M. \& Gras Pérez, M. E. (2009, noviembre). Las metodologías docentes y su valoración por parte de los estudiantes universitarios. Ponencia presentada en el II Congreso Internacional UNIVEST, Gerona.

Baruch, Y. (2006). Role-play Teaching. Acting in the Classroom. Management Learning, 37 (1), 43-61.

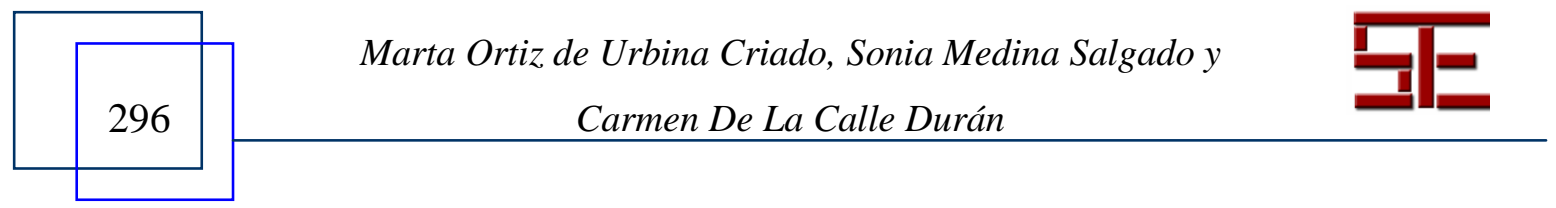




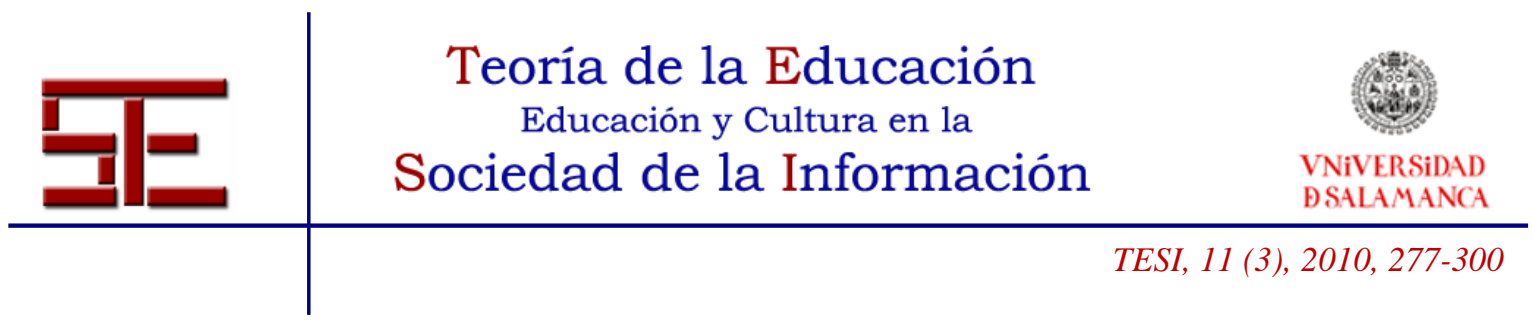

Beagly, T. M. (1998). Teaching about the Human Side of Corporate Restructuring: A Three-Stage Role-Play. Journal of Management Education, 22 (1), 70-84.

Brown, K .M. (1994). Using Role Play to Integrate Ethics into Business Curriculum. A Financial Management Example. Journal of Business Ethics, 13 (2), 105-110.

Buendía Eisman, L. \& Olmedo Moreno, E. (2000). Estrategias de aprendizaje y procesos de evaluación en la educación universitaria. Bordón, 52 (2), 151-163.

Cañeque, H. (1991). Juego y Vida. Buenos Aires: Ateneo.

Castelló, T. (2007). El disseny d'activitats facilitadores de l'aprenentatge. Girona: Institut de Ciències de 1'Educació Josep-Pallach de la UdG.

Chen, L. D., Muthitacharoen, A. \& Frolick, M. N. (2003). Investigating the Use of Role-Play Training to Improve the Communication Skills of IS Professional: Some Empirical Evidence. The Journal of Computer Systems, 43 (3), 67-73.

Crawford, C. (1982). The art of game design. Extraído el 26 de marzo de 2010 de http://www.vancouver.wsu.edu/fac/peabody/game-book/Coverpage.html.

De La Calle Durán, M. C. \& Ortiz-de-Urbina Criado, M. (2004). Fundamentos de Recursos Humanos. Madrid: Pearson-Prentice Hall.

De Miguel, M. (2006). Metodologías para optimizar el aprendizaje. Segundo objetivo del Espacio Europeo de Educación Superior. Revista Interuniversitaria de Formación del Profesorado, 20 (3), 71-91.

Inda Caro, M., Álvarez González, S. \& Álvarez Rubio, R. (2008). Métodos de evaluación en la enseñanza superior. Revista de Investigación Educativa, 26 (2), 539-552.

Garrido, F. J. \& Romero, J. J. (2008, septiembre). Simulación Virtual de la Praxis Jurídica: Proyecto de Innovación Docente "Role-playing en el Aprendizaje del Derecho”. Ponencia presentada en el I Congreso de Innovación Docente Universitaria. Régimen Jurídico. Didáctica e Innovación Docente. Granada.

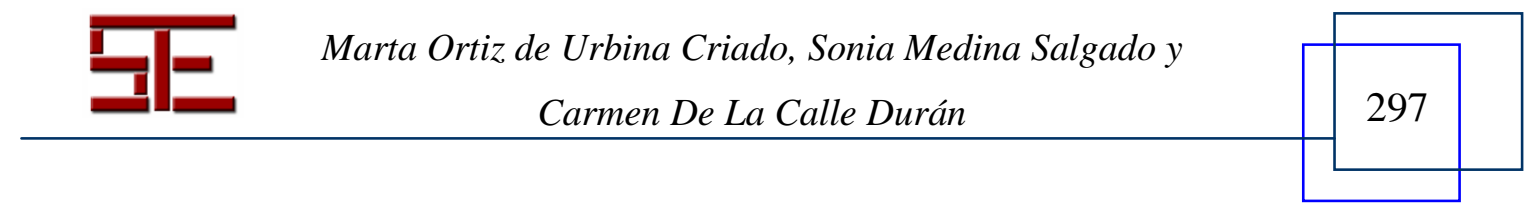




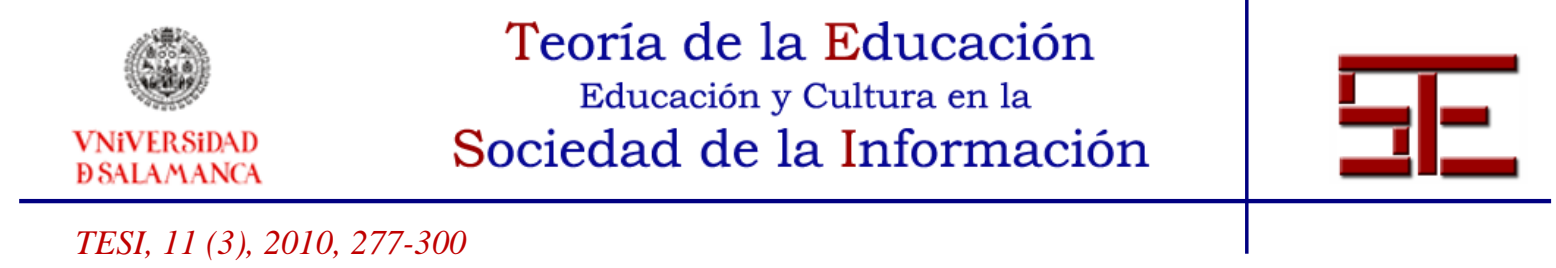

Gros, B. (2000). La dimensión socioeducativa de los videojuegos. Edutec. Revista Electrónica de Tecnología Educativa, 12. Extraído el 26 de marzo de 2010 de http://edutec.rediris.es/Revelec2/Revelec12/gros.pdf.

Hughes, R. L., Ginnett, R. C. \& Curphy, G. J. (1993). Leadership. Enhancing the Lessons of Experience. Homewood, Illinois: Irwin.

Ibáñez-Martín Mellado, J. A. (1990). Dimensiones de la competencia profesional del profesor de Universidad. Revista española de pedagogía, 48 (186), 239-280.

Jackson, P. T. \& Walters, J. P. (2000). Role-playing in Analytical Chemistry: The Alumni Speak. Journal of Chemical Education, 77 (8), 1019-1025.

Johnson, D. W. \& Johnson, R. T. (1994). Learning Together. En Sharan, S. (Ed.), Handbook of Cooperative Learning Methods. Connecticut: Greewood Press.

Jong, B., Chan, T., Wu, Y. \& Lin, T. (2006). Applying the adaptive learning material producing strategy to group learning. En First International Conference Edutainment. LNCS: Vol. 394 (pp: 39-49).

Khurana, R. (2007). From Higher Aims to Hired Hands: The Social Transformation of American Business Schools and the Unfulfilled Promise of Management as a Profession. Princeton University Press.

Lara, S. \& Rivas, S. (2009). Aprendizaje Autorregulado y Fomento de Competencias en Dos Asignaturas de Master a través del Empleo de Plantillas de Evaluación, Método del Caso, Role-playing y Vídeo Digital. Revista de Educación XXI, 12, 67-96.

Latorre, L. (2003). Juego y educación. Madrid, España: Comunidad de Madrid. Consejería de Educación, Dirección General de Promoción Educativa.

Marcano, B. (2008). Juegos Serios y Entrenamiento en la Sociedad Digital. En F.J. Sánchez i Peris (coord.), Videojuegos: una herramienta educativa del "homo digitalis" [monográfico en línea]. Revista Electrónica Teoría de la Educación. Educación y Cultura en la Sociedad de la Información, 9 (3), 93-107. Extraído el

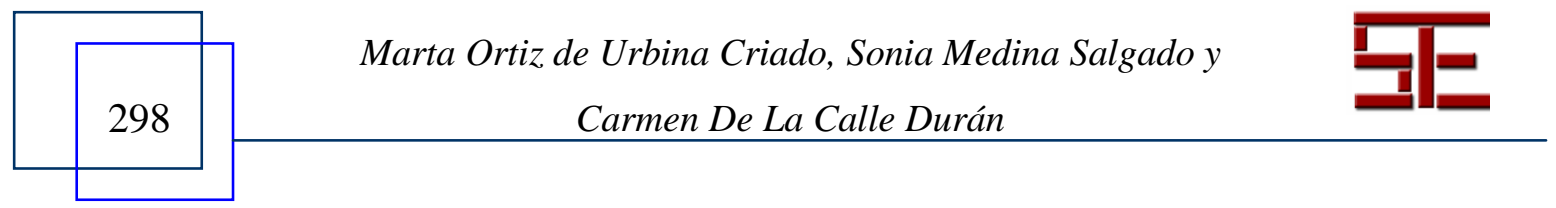




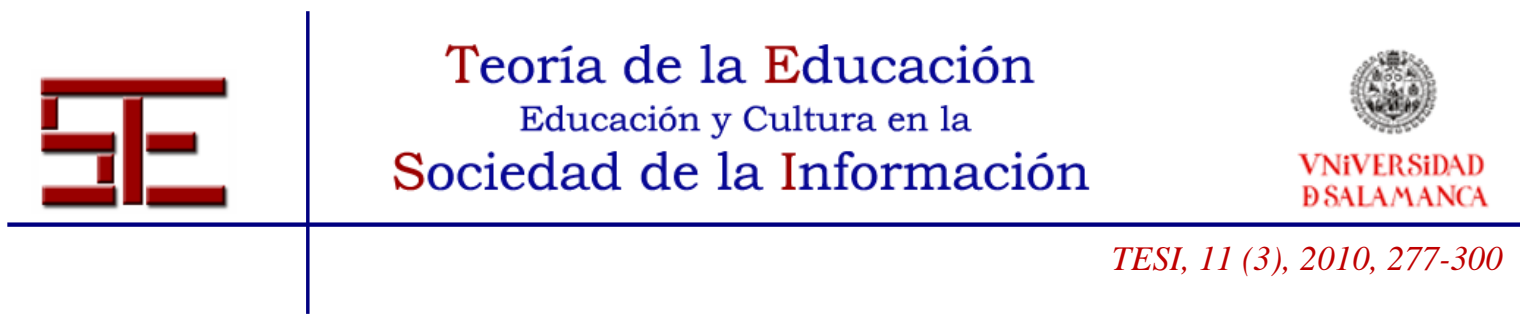

26 de marzo de 2010 de http://www.usal.es/ teoriaeducacion/rev_numero_09_03/n9_03_marcano.pdf.

Marzano, R. J. (1991). Creating an educational paradigm centred on learning through teacher-directed, naturalistic inquiry. En L. Idol \& B. F. Jones (eds.), Educational values and cognitive instruction. Implication for reform (pp. 411-442). Hillsdale Eribaum.

McDonald-Mann, D. G. (1998). Skill-based Training en The Center for Creative Leadership Handbook of Leadership Development. En C.D. Mccauley, R.S. Moxley \& E. V. Velsor (eds.), The Center for Creative Leadership Handbook of Leadership Development (pp. 106-126). San Francisco: Jossey-bass.

Ments, M. V. (1998). The Effective Use of Role-play: A Handbook for Teachers and Trainers. London: Kogan Page.

Muñoz, C. \& Huser, A. (2008). Experiential and Cooperative Learning: Using a Situation Analysis Project in Principles Marketing. Journal of Education for Business, 83 (4), 214-220.

Padilla Zea, N., González Sánchez, J. L., Gutiérrez, F. L., Cabrera, M. \& Paderewski, P. (2008). Diseño de Videojuegos Colaborativos y Educativos Centrados en la Jugabilidad. En A. B. Gil González, J. A. Velázquez Iturbide \& F. J. García Peñalvo (Coord). X Simposio Internacional de Informática Educativa (SIIE) (pp. 459-464). Salamanca: Ediciones Universidad de Salamanca.

Parra, E. (2002). Elementos para la docencia universitaria. Medellín: Universidad Cooperativa de Colombia.

Paulson D. R. \& Faust, J. L. (2008). Active Learning for the College Classroom. Extraído en enero de 2010 de http://www.calstatela.edu/dept/chem/chem2/LACTE/Index.html.

Peterson, R. (1996). Experiential Techniques Impart Practical Skills. Marketing News, $30(9)$.

Piaget, J. \& Inhelder, B. (1971). Psicología del niño. Madrid: Ed. Morata.

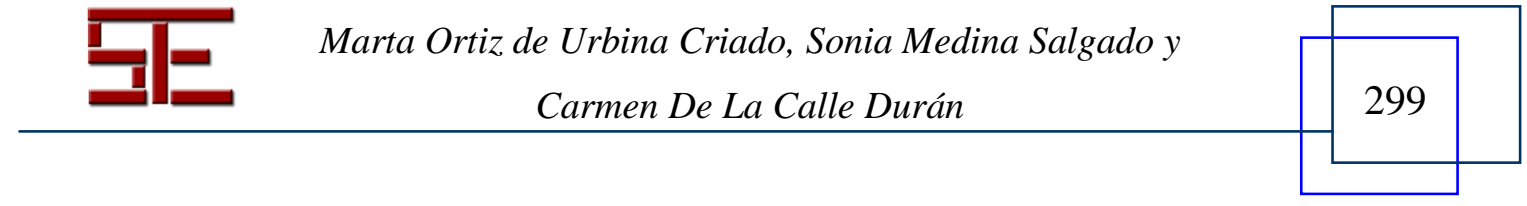




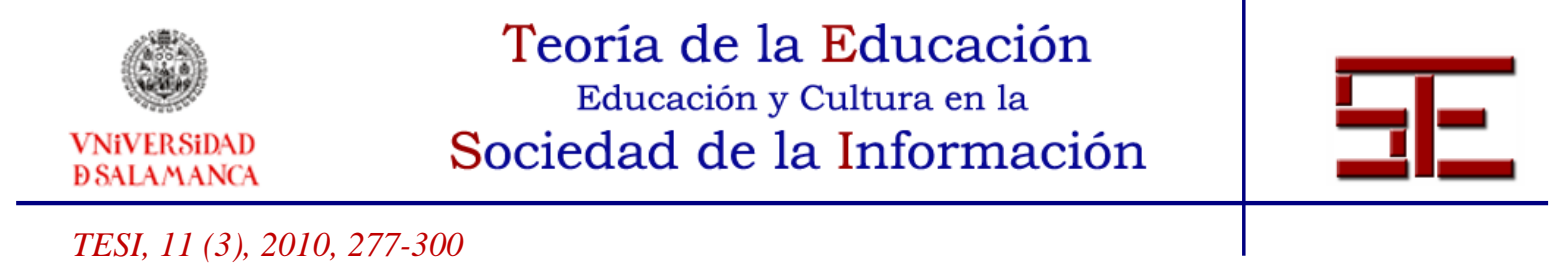

Rabinowitz, F. E. (1997). Teaching Counselling trough Semester-long Role play. Counsellor Education and Supervision, 36 (3), 216-223.

Rojas Ruiz, G. (2003). Estrategia para Fomentar Actitudes Interculturales Positivas en el Aula. Aldaba, 29, 71-88.

Sogunro, O. A. (2004). Efficacy of Role-playing Pedagogy in Training Leaders: Some Reflections. Journal of Management Development, 23 (3/4), 355-371.

Trigo Aza, E. (2006). Juego y Creatividad: El Re-Descubrimiento de lo Lúdico. En $V$ Congreso Internacional de Actividades Físicas Cooperativas. Oleiros-A Coruña, julio 2006.

Valiño, G. (2002). La Relación Juego y Escuela: Aportes Teóricos para su Comprensión y Promoción. Revista Conceptos, 77 (2).

Venable, B. B. (2001). Using Role-Play to Teach and Earn Aesthetics. Art Education, 54 (1), 47-51.

Waters, E., Woods, P. \& Noel, S. (1992). Role play: A versatile cooperative learning activity. Contemporary Education, 63 (3), 216-218.

Wood, C. M. (2003). The Effects of Creating Psychological Ownership among Students in Group projects. Journal of Marketing Education, 25, 241-249.

Yazici, H. J. (2004). Student Perceptions of Collaborative Learning in Operations Management Classes. Journal of Education for Business, 80 (2), 110-118.

\section{Notas:}

1. El concepto de educación democrática no es algo nuevo dentro de la enseñanza: John Dewey en su libro Democracia y Educación (1916), Ferrer i Guardia con su Escuela Moderna en Barcelona (1908) y otros padres de las teorías pedagógicas vieron la necesidad de hacer partícipes a los alumnos de su proceso de formación (Inda et al., 2008).

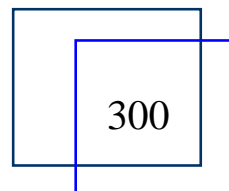

Marta Ortiz de Urbina Criado, Sonia Medina Salgado y Carmen De La Calle Durán 


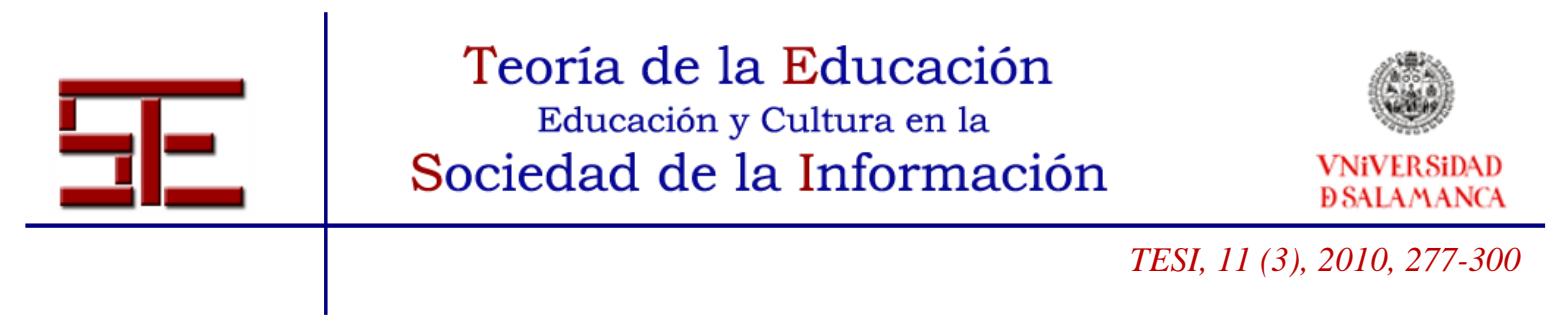

Para citar el presente artículo puede utilizar la siguiente referencia:

Ortiz-de-Urbina Criado, M., Medina Salgado, S. y De La Calle Durán, C. (2010): Herramientas para el aprendizaje colaborativo: una aplicación práctica del juego de rol, en Orejudo González, J.P. (Coord.) Perspectiva educativa y cultural de "juego de rol". Revista Teoría de la Educación: Educación y Cultura en la Sociedad de la Información. Vol. 11, no 3. Universidad de Salamanca, pp. 277-300 [Fecha de consulta: dd/mm/aaaa]. http://campus.usal.es/ revistas_trabajo/index.php/revistatesi/article/view/7463/7479

Marta Ortiz de Urbina Criado, Sonia Medina Salgado y 